Findings from a Preliminary Investigation of the Effects of Aquatic Habitat (Water) Availability on Giant Gartersnake (Thamnophis gigas) Demography and Reproduction in the Sacramento Valley, California, 2014-17

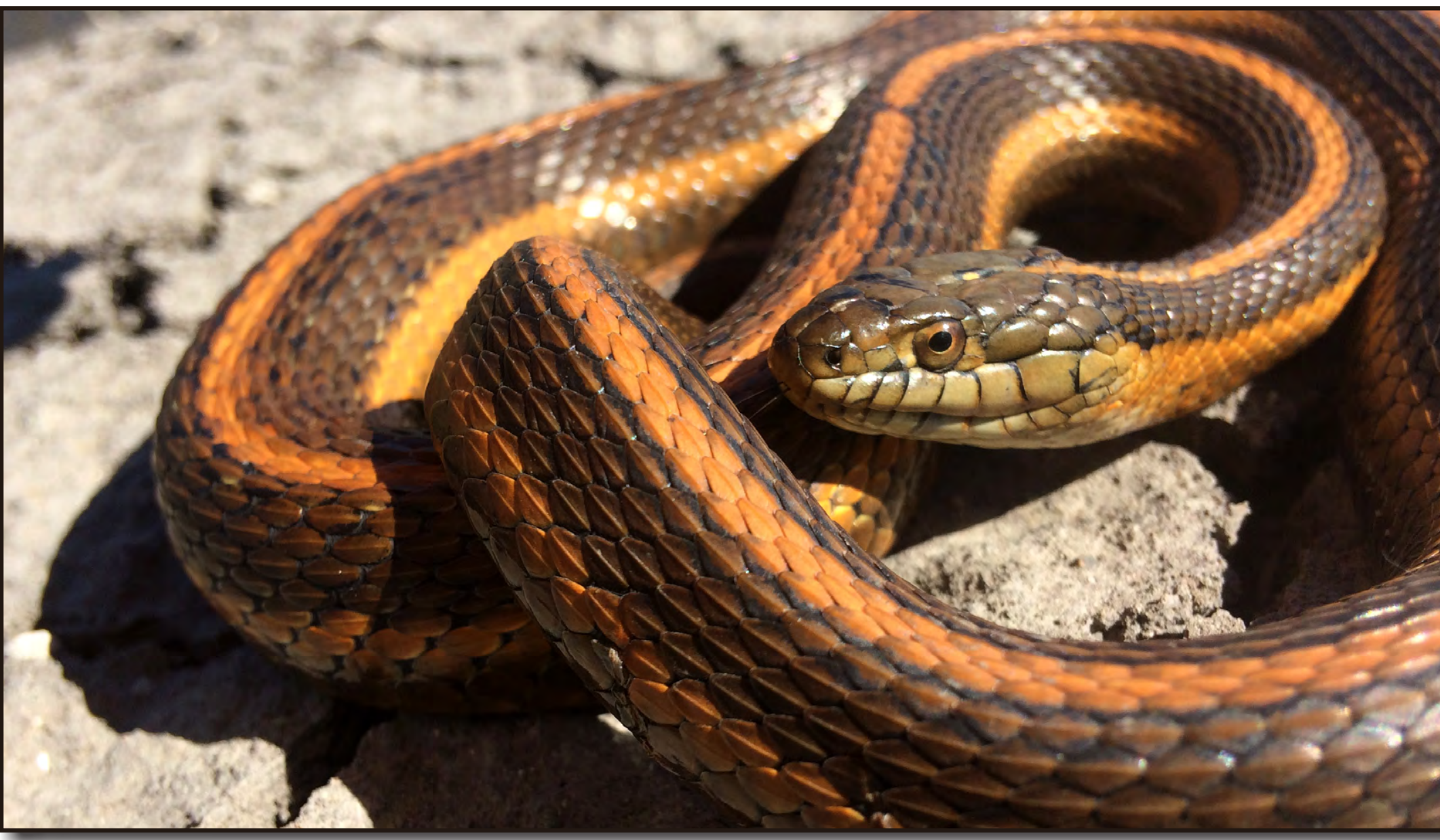

Open-File Report 2018-1114 
Cover image: Photograph showing a female giant gartersnake (Thamnophis gigas), Sacramento Valley, California. Photograph taken May 14th, 2018 by Zarina Sheikh, U.S. Geological Survey, Western Ecological Research Center. 


\section{Findings from a Preliminary Investigation of the Effects of Aquatic Habitat (Water) Availability on Giant Gartersnake (Thamnophis gigas) Demography and Reproduction in the Sacramento Valley, California, 2014-17}

By Jonathan P. Rose, Julia S.M. Ersan, Gabriel A. Reyes, K. Benjamin Gustafson, Alexandria M. Fulton, Kristen J. Fouts, Raymund F. Wack, Glenn D. Wylie, Michael L. Casazza, and Brian J. Halstead

Prepared in cooperation with the California Department of Water Resources

Open-File Report 2018-1114 


\section{U.S. Department of the Interior \\ RYAN K. ZINKE, Secretary}

U.S. Geological Survey
James F. Reilly II, Director

U.S. Geological Survey, Reston, Virginia: 2018

For more information on the USGS—-the Federal source for science about the Earth, its natural and living resources, natural hazards, and the environment-visit https://www.usgs.gov/ or call 1-888-ASK-USGS (1-888-275-8747).

For an overview of USGS information products, including maps, imagery, and publications, visit https:/store.usgs.gov.

Any use of trade, firm, or product names is for descriptive purposes only and does not imply endorsement by the U.S. Government.

Although this information product, for the most part, is in the public domain, it also may contain copyrighted materials as noted in the text. Permission to reproduce copyrighted items must be secured from the copyright owner.

Suggested citation:

Rose, J.P., Ersan, J.S.M., Reyes, G.A., Gustafson, K.B., Fulton, A.M., Fouts, K.J., Wack, R.F., Wylie, G.D., Casazza, M.L., and Halstead, B.J., 2018, Findings from a preliminary investigation of the effects of aquatic habitat (water) availability on giant gartersnake (Thamnophis gigas) demography in the Sacramento Valley, California, 2014-17: U.S. Geological Survey Open-File Report 2018-1114, 48 p.,

https://doi.org/10.3133/ofr20181114.

ISSN 2331-1258 (online) 


\section{Acknowledgments}

We thank the California Department of Water Resources for supporting this project. We also thank several cooperative landowners for access to the study sites. We also thank the giant gartersnake Technical Review Committee for providing feedback and guidance for this study, and two anonymous reviewers for their thorough reviews of this report. We thank Lisa Parker for administrative support. This study would not have been possible without the work of numerous biological technicians who collected field data on giant gartersnakes for the U.S. Geological Survey for many years. This work was performed under Institutional Animal Care and Use Committee protocol WERC-2014-01 and as stipulated in California Department of Fish and Wildlife Scientific Collecting Permit 10779 and U.S. Fish and Wildlife Service Recovery Permits TE-020548-5 and TE-157216-2. 


\section{Contents}

Acknowledgments .......................................................................................................................

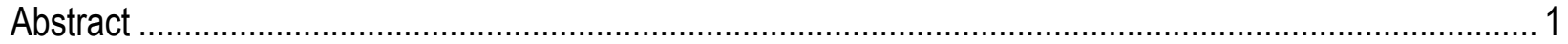

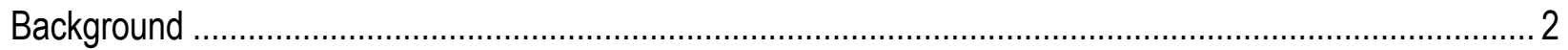

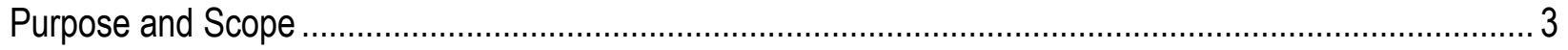

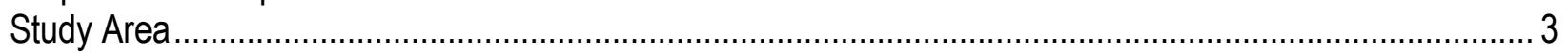

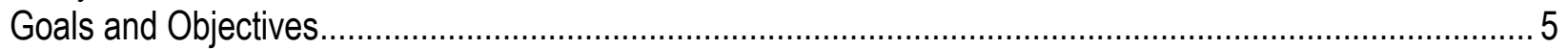

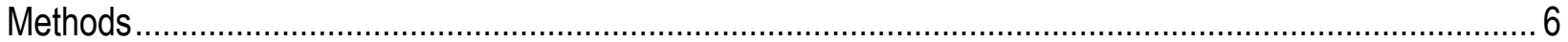

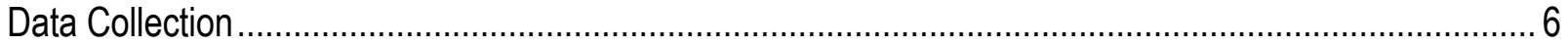

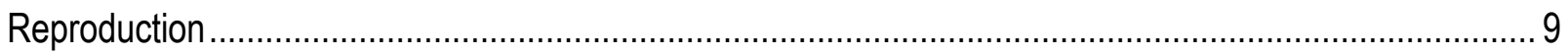

Health Assessments............................................................................................................... 9

Abundance, Population Growth, Capture Probability, and Survival Estimation ..................................... 9

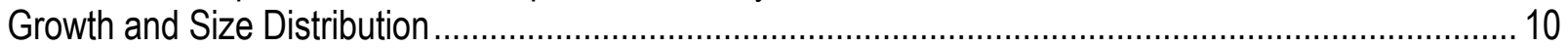

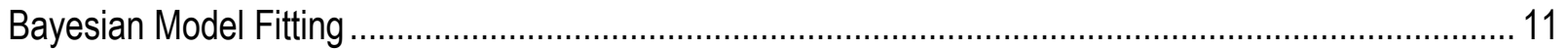

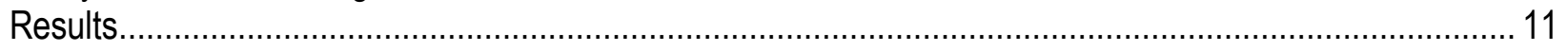

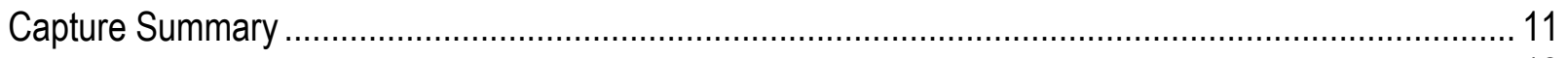

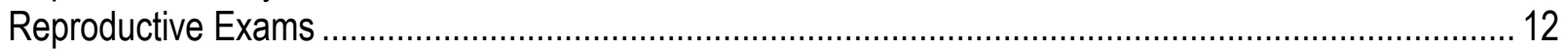

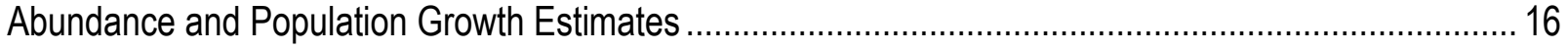

Annual Apparent Survival Estimates ........................................................................................ 24

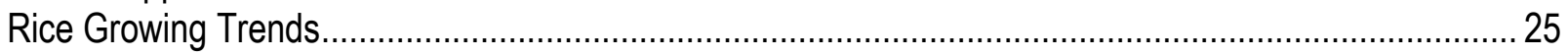

Size-Distribution of Captured Snakes..................................................................................... 27

Growth

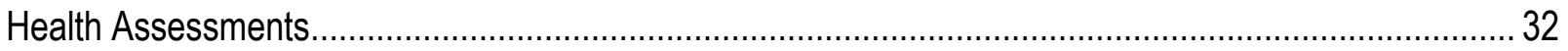

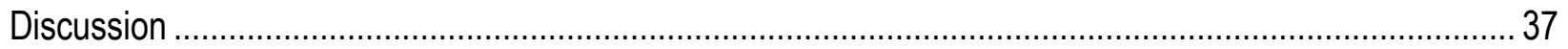

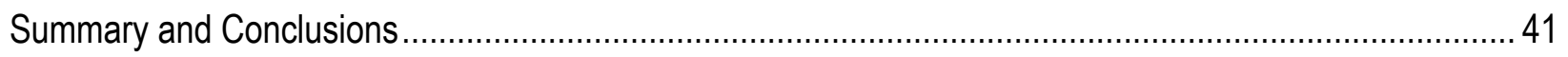

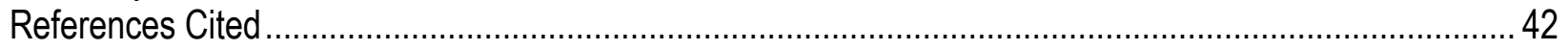

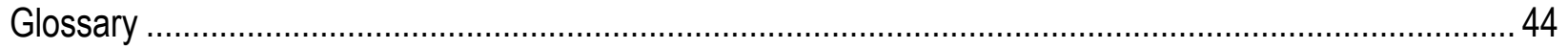

Appendix A. Details of Bayesian Models ....................................................................................... 46 


\section{Figures}

Figure 1. Location of eight sites sampled for giant gartersnakes in the Sacramento Valley, California, 2014-17.

Figure 2 . The probability a female giant gartersnake was gravid (a) and the observed litter size of gravid females (b) versus female snout-vent length (SVL) .....

Figure 3. Site-specific estimates for the mean probability a female giant gartersnake was gravid (a) and mean litter size (b) for each site in the Sacramento Valley, California

Figure 4. Estimated abundance $(\mathrm{N})$ of giant gartersnakes at each site in the Sacramento Valley, California, from 2014-17

Figure 5. Estimated population growth rate $(\lambda)$ of giant gartersnakes for 2014-15 and 2016-17 from eight sites in the Sacramento Valley, California.

Figure 6. Estimated daily capture probability (p) of giant gartersnakes from 2014-17 for eight sites in the Sacramento Valley, California.

Figure 7. Estimated annual capture probability ( $\mathrm{p}^{*}$ ) of giant gartersnakes from 2014-17 for eight sites in the Sacramento Valley, California

Figure 8. Estimated annual apparent survival $(\phi)$ of giant gartersnakes from 2014-17 for eight sites in the Sacramento Valley, California....

Figure 9. Proportional area of rice growing from 2014-17 at eight sites in the Sacramento Valley, California

Figure 10. Distribution of snout-vent lengths (SVL) of captured giant gartersnakes by site for eight sites in the Sacramento Valley, California, from 2014-17.

Figure 11. Distribution of the mass $(\mathrm{g})$ of captured giant gartersnakes from

eight sites in the Sacramento Valley, California, from 2014-17.

\section{Tables}

Table 1. Sampling effort and captures of giant gartersnakes in the Sacramento Valley, California, by site from 2014-17

Table 2. Frequency of year-over-year recaptures of giant gartersnakes by site in the

Sacramento Valley, California, from 2014-17

Table 3. Summary of reproductive data of giant gartersnakes collected from the Sacramento Valley, California, 2014-17, by site and year ....

Table 4. Giant gartersnake abundance estimates $(\mathrm{N})$ by site and year for eight study sites in the Sacramento Valley, California, 2014-17.

Table 5. Giant gartersnake daily capture probability estimates ( $\mathrm{p}$ ) by site and year for eight study sites in the Sacramento Valley, California, 2014-17 .

Table 6. Giant gartersnake annual capture probability estimates ( $\left.\mathrm{p}^{*}\right)$ by site and year for eight study sites in the Sacramento Valley, California, 2014-17

Table 7. Giant gartersnake annual apparent survival estimates $(\phi)$ by site and year for eight study sites in the Sacramento Valley, California, 2014-17 . 
Table 8. Extent of rice growing and other land cover types within a 1-km buffer of eight study sites in the Sacramento Valley, California, from 2014-17

Table 9. Parameter estimates from a von Bertalanffy growth model fit to growth data from giant gartersnakes at eight study sites sampled in the Sacramento Valley, 2014-17

Table 10. Growth rates of giant gartersnakes from 2014-17 summarized by site, sex, and year, for eight sites in the Sacramento Valley, California.

Table 11. Summary of blood cell counts, hemoglobin, and hematocrit of female giant gartersnakes of reproductive size by site and year for eight sites in the Sacramento Valley, California, 2014-17.

Table 12. Summary of blood solutes of female giant gartersnakes of reproductive size by site and year for eight sites in the Sacramento Valley, California, 2014-17

\section{Conversion Factors}

International System of Units to U.S. customary units

\begin{tabular}{|c|c|c|}
\hline Multiply & By & To obtain \\
\hline \multicolumn{3}{|c|}{ Length } \\
\hline millimeter (mm) & 0.0393701 & inch (in.) \\
\hline kilometer (km) & 0.621371 & mile $(\mathrm{mi})$ \\
\hline \multicolumn{3}{|c|}{ Mass } \\
\hline milligram (mg) & 0.000035 & ounce, avoirdupois (oz) \\
\hline $\operatorname{gram}(\mathrm{g})$ & 0.035274 & ounce, avoirdupois (oz) \\
\hline \multicolumn{3}{|c|}{ Volume } \\
\hline milliliter $(\mathrm{mL})$ & 0.0338 & ounce, fluid (fl. oz) \\
\hline liter (L) & 1.05669 & quart (qt) \\
\hline
\end{tabular}

\section{Abbreviations}

AST

CMR

CRI

JAGS

MCMC

PIT

$\mathrm{Pr}$

SD

SVL

USGS aspartate aminotransferase

capture-mark-recapture

credible interval

Just Another Gibbs Sampler

Markov Chain Monte Carlo

passive integrated transponder

probability

standard deviation

snout-vent length

U.S. Geological Survey 


\title{
Findings from a Preliminary Investigation of the Effects of Aquatic Habitat (Water) Availability on Giant Gartersnake (Thamnophis gigas) Demography and Reproduction in the Sacramento Valley, California, 2014-17
}

By Jonathan P. Rose, ${ }^{1}$ Julia S.M. Ersan, ${ }^{1}$ Gabriel A. Reyes, ${ }^{1}$ K. Benjamin Gustafson, ${ }^{1}$

Alexandria M. Fulton, ${ }^{1}$ Kristen J. Fouts, ${ }^{1}$ Raymund F. Wack, ${ }^{2}$ Glenn D. Wylie, ${ }^{1}$ Michael L. Casazza, ${ }^{1}$ and Brian J. Halstead ${ }^{1}$

\begin{abstract}
The giant gartersnake (Thamnophis gigas) is a semi-aquatic species of snake precinctive to the Central Valley of California. Because the Central Valley has experienced a substantial loss of wetland habitat, giant gartersnake populations are largely found in aquatic habitats associated with rice agriculture. In dry years, less water may be available for rice agriculture, resulting in less aquatic habitat, which could have cascading effects on giant gartersnake populations. We present 2 years of data intended to examine how the demography of giant gartersnakes is affected by the availability of aquatic habitat on the landscape (2016-17), along with 2 years of (sparse) preliminary data (2014-15) collected as part of an earlier radio-telemetry study on giant gartersnake movement behavior. We sampled agricultural canals near rice fields for giant gartersnakes at 8 sites distributed throughout the Sacramento Valley. Five sites were sampled from 2014-17, and 3 sites were sampled from 2015-17. In total, we made 2,995 captures of 1,011 snakes from 2014-17. We used these capture data to fit a multi-site Jolly-Seber model to estimate the abundance of giant gartersnakes as well as the daily and annual probability of capture at each site. We used remotely sensed Landsat data to characterize the extent of flooded rice fields surrounding each site in each year. In addition, we collected 175 females from 201417 and delivered them to the Sacramento Zoo for health assessments and reproductive exams.
\end{abstract}

${ }^{1}$ U.S. Geological Survey

${ }^{2}$ Sacramento Zoo; University of California, Davis 
The abundance of giant gartersnakes varied among sites, and abundance estimates were more precise in 2016 and 2017 when sampling effort was greatest. The probability of a giant gartersnake being captured at least once in a year was higher in 2016 and 2017 than 2014 and 2015, and recaptures of snakes marked the previous year were highest in 2016 and 2017 as well. Mean annual apparent survival was estimated to be 0.40 but varied among sites from a low of 0.14 to a high of 0.63 . Five sites had diverse size distributions that included abundant sub-adult and large adult female snakes. One site had a truncated size distribution with few large adult female snakes, and 2 sites had mostly large adult-sized snakes and few small individuals. Both the probability a female was gravid and a female's litter size were positively related to the female's snout-vent length. Somatic growth rates varied more among years than among sites, and females grew faster (in millimeters per day) than male snakes.

The proportion of the landscape around each site under active rice cultivation fluctuated over time (generally between 60-90 percent of the landscape was active rice growing, although this proportion was lower for some sites in some years), and variation in rice growing was asynchronous among sites. This study demonstrates that intensive demographic sampling enables estimation of several key demographic variables at each study site. Continued sampling would allow for investigating potential relationships between the amount of rice growing at a site and demographic parameters such as growth, survival, and reproduction.

\section{Background}

The California Department of Water Resources (DWR) has a mission to manage and protect California's water resources for both human and natural environments. Supplying sufficient water to meet the demands of urban, residential, agricultural, and industrial users is a challenge in California because much of the state has a Mediterranean or desert climate in which precipitation is limited through much of the year. In the Sacramento Valley (northern Central Valley), the availability of water is a critical component of both agricultural production and the preservation of wildlife species that are dependent on wetland habitats. The giant gartersnake (Thamnophis gigas) is a semi-aquatic species of snake precinctive to the Central Valley of California, where it inhabits wetlands, marshes, and canals associated with rice agriculture (Halstead and others, 2014). Giant gartersnakes are dependent on wetland habitat throughout their spring and summer active season (Halstead and others, 2015b). The Central Valley has lost over 90 percent of its wetland habitat since the 19th century (Frayer and others, 1989), and consequently, the giant gartersnake is now listed as a threatened species under the State (Fish and Game Commission, 1971) and Federal (U.S. Fish and Wildlife Service, 1993) Endangered Species Acts. Giant gartersnakes now occur primarily in habitats associated with rice agriculture (Halstead and others, 2010) because the process of growing rice creates flooded fields and canals that approximate the marsh habitat needed by giant gartersnakes. 
Activities that influence the availability of water for rice agriculture have the potential to affect threatened giant gartersnakes (Reyes and others, 2017). Rice farmers can respond to dry years by leaving some rice fields idle and transferring their water to other users downstream. This helps ensure a reliable water supply for other users, but leaving rice fields idle reduces the amount of aquatic habitat available for wildlife on the landscape. It remains unknown how such water transfers and annual changes in the extent of wetland habitat affect population growth rates and demographic vital rates such as reproduction, survival, and somatic growth in giant gartersnake populations. This study seeks to understand how the availability of aquatic habitat influences the health of individual giant gartersnakes and the demography of giant gartersnake populations.

\section{Purpose and Scope}

This report summarizes the methods and findings of a study conducted by the U.S. Geological Survey, in cooperation with the California Department of Water Resources, to investigate the effect of the availability of aquatic habitat on the demography of giant gartersnakes inhabiting rice growing areas in the Sacramento Valley, California. The report presents estimates of the abundance, somatic growth, fecundity, and survival of giant gartersnakes from eight sites in the Sacramento Valley studied from 2014-17. The report also presents data on the area of rice growing at each of the eight sites from 2014-17. Throughout this report, words defined in the glossary are presented in bold font upon their first use.

\section{Study Area}

The data used in this report come from eight study sites distributed throughout the Sacramento Valley of California in Butte, Colusa, Glenn, Sacramento, Sutter, and Yolo Counties (fig. 1). 


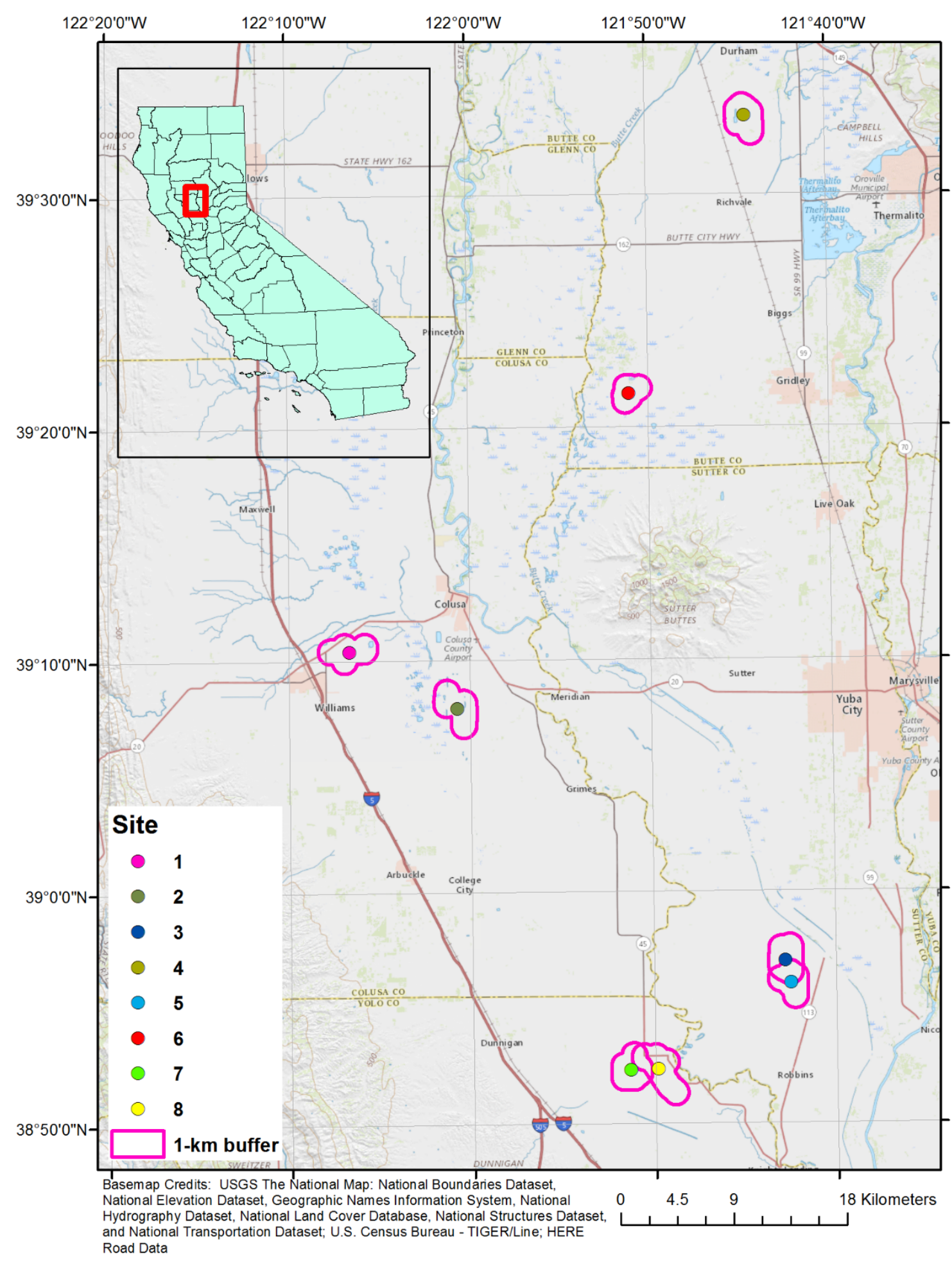

Figure 1. Location of eight sites sampled for giant gartersnakes in the Sacramento Valley, California, 2014-17. Each circle represents the average Universal Transverse Mercator (UTM) coordinates for the traps located at that site. 


\section{Goals and Objectives}

The goal of this study is to measure how the availability of aquatic habitat (chiefly rice fields and their associated canals) affects the demography of giant gartersnake populations and the reproductive biology of female giant gartersnakes. To meet this goal, our objectives are to measure the following aspects of the biology of giant gartersnakes:

1. Reproductive traits of female giant gartersnakes.

2. The survival rate of giant gartersnakes.

3. The abundance of giant gartersnakes.

4. The growth rate of giant gartersnake populations.

5. The somatic growth rate (growth in body length) of individual giant gartersnakes.

6. The health of individual giant gartersnakes.

In addition, we measure the availability of aquatic habitat at each site, in each year, by observing the proportion of the landscape undergoing active rice cultivation. At our study sites, rice fields and their associated canals provide the only aquatic habitat for giant gartersnakes. This information will be vital for modeling the effect of water transfers and rice idling on the demography of giant gartersnake populations. The demographic information collected in this study could be used to project the effect that water management activities will have on the persistence of giant gartersnake populations in the Sacramento Valley. This report presents a summary of the data collected from 8 populations of giant gartersnakes inhabiting canals associated with rice agriculture from 2014-17. This period includes 2 years of preliminary sampling as part of an earlier radio-telemetry study (2014-15; Reyes and others, 2017) and the first 2 years (2016-17) of a planned 6-year intensive demographic study. For reasons out of our control, the demographic study was discontinued prior to field sampling in early 2018 , and this report is intended to present the preliminary results from the first 2 years of data collection. We present estimates of demographic parameters and the amount of rice growing at each site for the period from 2014-17. Because of the short time series of data available, and the limited sampling that took place in 2014 and 2015, we refrain from using the proportion of rice grown at a site each year as a covariate of demographic and health parameters. Instead, we present the proportion of rice grown at each site as an indication of the variation among sites over time that we might expect to observe in a long-term study. If more data are collected in the coming years, we could obtain more precise estimates of the demographic parameters of interest and investigate how the variation in rice growing over time affects giant gartersnake health, reproduction, somatic growth, survival, and population growth. 


\section{Methods}

\section{Data Collection}

We sampled 5 sites for giant gartersnakes from 2014-17, and 3 sites from 2015-17 (fig. 1; table 1). Each site comprises canals surrounded by agricultural fields. We captured giant gartersnakes by setting aquatic funnel traps along the edges of canals and emergent vegetation. We also opportunistically captured snakes by hand while setting and checking traps. We did not bait traps, and traps were placed so that the surface of the water was approximately at the midpoint of the funnel opening (Casazza and others, 2000; Halstead and others, 2013). In 2014 and 2015, we set 100-200 traps per site, and in 2016 and 2017, we set a total of 250 traps per site each year. Sites were trapped more intensively in 2016 and 2017 because the objective of the study was to estimate abundance and survival using capture-mark-recapture data. The traplines also covered a larger area at each site in the latter 2 years to help reduce the influence of emigration on capture-mark-recapture estimates of apparent survival. Sites were sampled less intensively in 2014 and 2015 because the primary objective was to capture snakes to implant radio-transmitters as part of a study of giant gartersnake spatial ecology and movement (Reyes and others, 2017). We measured snout-vent length (SVL) by straightening snakes on a meter stick. We weighed snakes using a Pesola ${ }^{\circledR}$ spring scale. We determined the sex of all captured snakes through cloacal probing. We marked each snake by using a medical cautery device to brand a unique combination of ventral scales that corresponds to a numerical identifier (Winne and others, 2006) and also injected Passive Integrated Transponders (PIT) subcutaneously into snakes $>30$ grams $(\mathrm{g})$ mass using a syringe injector.

From 2014-17, we also collected females from each site and took them to the Sacramento Zoo, where trained veterinarians examined their reproductive status using Xradiography and gave each snake a full health assessment. Snakes were removed from the wild for up to 7 days and were returned to their site of capture after the completion of health assessments and X-rays. A veterinarian drew 1.0 milliliters $(\mathrm{mL})$ of blood from the ventral tail vein for analysis of plasma biochemistry and hematological parameters. These parameters can be evaluated to see if snakes exhibit signs of stress or infection (Wack and others, 2012). Hematological parameters included red blood cell count, hemoglobin (grams per deciliter), packed cell volume (the percent of total blood volume made up of red blood cells), and plasma protein (grams per deciliter). We also counted white blood cells and further classified white blood cells into one of 6 types: azurophils, basophils, eosinophils, heterophils, lymphocytes, and monocytes. Elevated white blood cell counts can indicate the presence of infection, whereas low white blood cell counts could indicate a weakened immune system (Reyes and others, 2017). To determine pack cell volume, we centrifuged microhematocrit tubes for 10 minutes and measured the proportion of the sample comprising red blood cells. We employed a modified azidemethemoglobulin reaction, following Wack and others (2012), to measure hemoglobin. We used a handheld refractometer (JorVet model J351, Jorgensen Laboratories Inc., Loveland, Colorado) to measure plasma protein. Within 3 hours of blood collection, we used the unipette method and a hemocytometer to manually count red blood cells and white blood cells (Campbell and Ellis, 2007). To measure the proportion of white blood cell types, we stained a blood smear and counted at least 100 white blood cells under $1,000 \times$ magnification. 
Table 1. Sampling effort and captures of giant gartersnakes in the Sacramento Valley, California, by site from 2014-17.

[-- indicates a site was not sampled]

\begin{tabular}{|c|c|c|c|c|c|c|c|}
\hline Year & Site & Start date & End date & Days & Trap-days & Snakes & Captures \\
\hline \multirow[t]{8}{*}{2014} & 1 & $5 / 14 / 2014$ & $6 / 5 / 2014$ & 21 & 1,998 & 41 & 76 \\
\hline & 2 & $6 / 27 / 2014$ & $9 / 2 / 2014$ & 64 & 5,747 & 15 & 20 \\
\hline & 3 & $5 / 13 / 2014$ & $7 / 16 / 2014$ & 59 & 5,599 & 27 & 36 \\
\hline & 4 & -- & -- & -- & -- & -- & -- \\
\hline & 5 & $6 / 13 / 2014$ & $7 / 23 / 2014$ & 37 & 3,800 & 10 & 13 \\
\hline & 6 & -- & -- & -- & -- & -- & -- \\
\hline & 7 & -- & -- & -- & -- & -- & -- \\
\hline & 8 & $5 / 19 / 2014$ & $6 / 11 / 2014$ & 20 & 1,996 & 31 & 40 \\
\hline \multirow[t]{8}{*}{2015} & 1 & $4 / 21 / 2015$ & $7 / 10 / 2015$ & 75 & 7,521 & 25 & 41 \\
\hline & 2 & $4 / 22 / 2015$ & $4 / 23 / 2015$ & 1 & 100 & 7 & 8 \\
\hline & 3 & $4 / 28 / 2015$ & $9 / 1 / 2015$ & 121 & 4,020 & 23 & 37 \\
\hline & 4 & $5 / 1 / 2015$ & $6 / 21 / 2015$ & 48 & 4,893 & 20 & 36 \\
\hline & 5 & $4 / 26 / 2015$ & $8 / 28 / 2015$ & 119 & 9,438 & 11 & 15 \\
\hline & 6 & $5 / 30 / 2015$ & $8 / 31 / 2015$ & 90 & 6,900 & 31 & 76 \\
\hline & 7 & $4 / 24 / 2015$ & $8 / 24 / 2015$ & 117 & 9,474 & 15 & 25 \\
\hline & 8 & $4 / 23 / 2015$ & $4 / 27 / 2015$ & 4 & 400 & 6 & 6 \\
\hline \multirow{8}{*}{2016} & 1 & $4 / 24 / 2016$ & $7 / 18 / 2016$ & 83 & 17,613 & 73 & 189 \\
\hline & 2 & $4 / 28 / 2016$ & $7 / 22 / 2016$ & 83 & 19,302 & 88 & 267 \\
\hline & 3 & $4 / 25 / 2016$ & $7 / 17 / 2016$ & 81 & 19,862 & 75 & 216 \\
\hline & 4 & $5 / 6 / 2016$ & $7 / 16 / 2016$ & 69 & 15,438 & 55 & 123 \\
\hline & 5 & $5 / 4 / 2016$ & $8 / 6 / 2016$ & 92 & 17,458 & 14 & 29 \\
\hline & 6 & $4 / 27 / 2016$ & $7 / 26 / 2016$ & 88 & 18,317 & 81 & 245 \\
\hline & 7 & $5 / 2 / 2016$ & $7 / 27 / 2016$ & 84 & 13,811 & 27 & 60 \\
\hline & 8 & $4 / 19 / 2016$ & $7 / 16 / 2016$ & 86 & 19,700 & 127 & 438 \\
\hline \multirow[t]{8}{*}{2017} & 1 & $4 / 25 / 2017$ & $7 / 15 / 2017$ & 77 & 16,307 & 68 & 203 \\
\hline & 2 & $4 / 26 / 2017$ & $7 / 15 / 2017$ & 76 & 17,485 & 59 & 124 \\
\hline & 3 & $5 / 2 / 2017$ & $7 / 15 / 2017$ & 70 & 15,605 & 55 & 106 \\
\hline & 4 & $6 / 3 / 2017$ & $7 / 22 / 2017$ & 46 & 10,275 & 43 & 130 \\
\hline & 5 & $5 / 10 / 2017$ & $7 / 15 / 2017$ & 62 & 13,092 & 22 & 43 \\
\hline & 6 & $5 / 8 / 2017$ & $7 / 15 / 2017$ & 64 & 13,616 & 41 & 73 \\
\hline & 7 & $5 / 31 / 2017$ & $7 / 22 / 2017$ & 50 & 11,359 & 12 & 26 \\
\hline & 8 & $4 / 22 / 2017$ & $7 / 15 / 2017$ & 80 & 19,487 & 123 & 294 \\
\hline
\end{tabular}


We measured a suite of plasma biochemistry parameters, including AST (aspartate aminotransferase; international units per liter [IU/L]), creatinine kinase (IU/L), uric acid (milligrams per deciliter $[\mathrm{mg} / \mathrm{dL}])$, glucose $(\mathrm{mg} / \mathrm{dL})$, calcium $(\mathrm{mg} / \mathrm{dL})$, phosphorus $(\mathrm{mg} / \mathrm{dL})$, total protein (grams per deciliter $[\mathrm{g} / \mathrm{dL}])$, albumin $(\mathrm{g} / \mathrm{dL})$, globulin $(\mathrm{g} / \mathrm{dL})$, potassium (millimoles per deciliter $[\mathrm{mmol} / \mathrm{dL}])$, and sodium $(\mathrm{mmol} / \mathrm{dL})$. To measure plasma biochemistry parameters, we began by centrifuging a heparinized blood microtainer tube for 10 minutes. We then used a VetScan analyzer (Abaxis North America, Union City, California) with an avian/reptile specific rotor (Avian/Reptilian Profile Plus) to measure these parameters. All plasma biochemistry analysis was performed within 30 minutes of the collection of the blood sample. High concentrations of plasma solutes such as phosphorus, sodium, and potassium can indicate signs of dehydration. Elevated concentrations of AST might be a sign that organs are diseased or damaged, and elevated creatinine kinase in blood plasma is a potential sign of damage to muscle tissue (Reyes and others, 2017).

Each female's reproductive status was determined by examining abdominal X-rays for the presence of enlarged eggs or developing embryos. If a female was gravid, the number of enlarged, ovulated eggs or developing embryos was counted, and we use these counts as an estimate of the litter size or number of offspring a female was carrying. From 2014-16, examined females were primarily individuals that had radio transmitters implanted for a previous study (Reyes and others, 2017). In 2017, we chose females $\geq 500$ millimeters (mm) SVL to receive health assessments and reproductive exams, with the exception of one female that measured $435 \mathrm{~mm}$ SVL. We did not selectively choose to examine females that appeared gravid; rather, we sought to examine a representative sample of females that were within the size range for reproductive maturity (Rose and others, 2018c). This sample allows the estimation of the proportion of females that are gravid that year and how body size (SVL) affects the probability a female is gravid.

To characterize the landscape at each site, we used land cover data within a 1-kilometer $(\mathrm{km})$ buffer of our 2017 trap locations. We decided on a 1-km buffer based on analysis of radiotelemetry data from these sites using the methods and data reported in Reyes and others (2017). We calculated the mean (across all snakes) of the maximum distance between pairs of observations of the same radio-tracked individual in the same calendar year, which was approximately $1 \mathrm{~km}$. In other words, on average, the largest distance between locations of a radio-tracked snake within one year was approximately $1 \mathrm{~km}$; therefore, snakes captured in traps would be expected to primarily inhabit the area within $1 \mathrm{~km}$ of the traplines. We classified remotely sensed Landsat 8 imagery into 3 different agricultural land cover types: active rice growing, fallow fields, and non-rice crops. All other land covers were classified as "other," and no other wetlands were found within the $1-\mathrm{km}$ buffer of the study sites. For more details on the method used to identify rice and other land cover types, please see the description in Reyes and others (2017) and Zhong and others (2016). Reyes and others (2017) compared the 2015 land cover classification from Landsat 8 imagery to the United States Department of Agriculture (USDA) "Crop Data Layer" from 2015 and found that the Landsat based tool classified rice with 96.3-percent accuracy. We also qualitatively compared the land cover classifications from Landsat 8 imagery in 2017 to ground-based surveys of fields and found good agreement at each site. For 2017, we were able to estimate the extent of active rice growing but could not estimate other land cover types. 


\section{Reproduction}

We tested for fixed effects of female SVL and the date of examination (linear and quadratic effects of day of year) on the probability a female was gravid using a Bayesian regression model with a binomial response ( 1 if gravid, 0 if not) and a random effect of site on the intercept. We tested for a fixed effect of female SVL on litter size using a Bayesian Poisson regression model (similar to a generalized linear model with a log link for the linear predictor) with litter size as the response and a random effect of site on the intercept. The random effects of site on the intercept for these models allow the response (either fecundity or the probability a female is gravid) to vary among sites, while still sharing information among sites to better estimate the relationship between the response and snake size. For example, we might expect the proportion of females that are gravid to vary among sites, owing to differences in the environment, and we want our model to account for that variation. We standardized female SVL and examination date to have a mean equal to zero and standard deviation of one and used these standardized values as covariates in our regression models. Prior probability distributions used in our Bayesian models of reproduction and fecundity are described in tables A1 and A2.

\section{Health Assessments}

We report summary statistics for several blood and plasma biochemistry parameters from 159 snakes that received health assessments from 2014-17. We qualitatively compare the values of these parameters to the baseline values from a sample of "apparently healthy" giant gartersnakes presented by Wack and others (2012). Because of the short time series of this study and the small sample sizes from each site from 2014-16, we do not test for or report trends in blood and plasma biochemistry parameters.

\section{Abundance, Population Growth, Capture Probability, and Survival Estimation}

We used the capture data from 2014-17 to estimate the abundance $(N)$, daily capture probability $(p)$, and the annual apparent survival rate $(\phi)$ of snakes at each study site in each year. The apparent survival rate is the probability of survival multiplied by the probability of an individual remaining at the site ( 1 - the probability of emigration). We fit a Bayesian multi-site Jolly-Seber capture-mark-recapture (CMR) model, based on models presented by Kéry and Schaub (2012) and Kéry and Royle (2016), to our capture data (see table A3 for details on prior distributions). We included random effects of site and year (nested within site) on capture probability and annual survival. These random effects account for variation among sites or among years that is not accounted for by other means. For example, we might expect survival to vary over time owing to fluctuations in environmental conditions, and the temporal random effect allows us to estimate that variation. We calculated the probability an individual was captured at least once in a year ("annual capture probability," $\hat{p}_{t}^{*}$ ) using eq. 1 , where $\hat{p}_{t}$ is the estimated daily capture probability in year $t$, and $k_{t}$ is the number of days a site was sampled in year $t$.

$$
\hat{p}_{t}^{*}=1-\left(1-\hat{p}_{t}\right)^{k_{t}}
$$


Based on our annual estimates of abundance for each site, we also estimate the population growth rate for year $t, \lambda_{\mathrm{t}}$, based on the estimated abundances in year $t\left(\widehat{N}_{t}\right)$ and year $t+1\left(\widehat{N}_{t+1}\right)$ (eq. 2).

$$
\hat{\lambda}_{t}=\frac{\widehat{N}_{t+1}}{\widehat{N}_{t}}
$$

Because our sampling intensity (number of trap-days) and the area sampled increased in 2016 and 2017 compared to 2014 and 2015, we only present estimates of $\lambda$ from 2014-15 and 2016-17. Estimates of $\lambda$ from 2015-16 are likely to be influenced by the apparent "immigration" of new individuals to the sampled population owing to the larger area sampled and longer sampling period in 2016 compared to 2015.

\section{Growth and Size Distribution}

We fit a Bayesian von Bertalanffy growth model to the SVL of snakes captured and measured two or more times. We followed the methods used in a previous study of giant gartersnake growth (Rose and others, 2018b) and included fixed effects of sex on asymptotic size (SVL; $\mathrm{L}_{\infty}$ ) and the von Bertalanffy growth coefficient $(k)$. The model also included random effects of site and year on $k$ and a random effect of site on $\mathrm{L}_{\infty}$. We use this model to estimate if sites differ in the average asymptotic SVL of snakes, and in $k$, the rate at which snakes grow to approach their asymptotic SVL. We used the same model and priors as in a previous study that fit a von Bertalanffy growth model to giant gartersnake growth data (table A4; Rose and others, 2018b).

As another method for characterizing variation in growth between the sexes and among sites and years, we calculated the daily somatic growth rate $(\mathrm{mm} /$ day) for snakes captured and measured in consecutive years. We averaged growth over the entire interval between captures, including the inactive season, and thus our growth rate estimates are averaged over both the inactive and active seasons. We included fixed effects of sex and SVL (at first capture), an interactive effect of sex and SVL on growth rate, and random effects of individual, site, and year to account for repeated measurements of the same individual and potential correlations of growth parameters within sites and years. We standardized SVL as described above and used the standardized SVL value as a covariate of growth rate. For our analysis of growth, and the distribution of snake sizes (SVL and mass) by site, we averaged the size measurements of snakes captured multiple times within the same year. We included all growth measurements, including apparent negative growth (shrinkage) between captures. 


\section{Bayesian Model Fitting}

We use Bayesian analysis in this study because of the flexibility it provides in designing statistical models tailored to our data. We fit all Bayesian models using the software Just Another Gibbs Sampler (JAGS; Plummer, 2003) to sample the posterior distribution using Markov Chain Monte Carlo (MCMC) algorithms. We accessed JAGS through the statistical program R (R Core Team, 2016) using the "runjags" package (Denwood, 2016). We used uninformative priors for parameters on the probability scale (uniform probability of any value between 0 and 1) and weakly informative priors for all other parameters (see tables A1-A4 for prior distributions). We used half-Cauchy priors for the standard deviation of all random effects (Gelman, 2006; Polson and Scott, 2012). We ran each model on five independent chains (except for the CMR model, which was run on four chains) for enough iterations that the posterior distributions appeared to have converged on a stationary distribution (see appendix A for details). We assessed the convergence of models by inspecting trace plots and calculating the Brooks-Gelman convergence diagnostic (Brooks and Gelman, 1998). No models showed evidence of poor convergence. For model-estimated parameters, we present the posterior mean and symmetric 95-percent credible interval (CRI), unless specified otherwise. To measure the strength of evidence for a parameter having a value above or below a threshold (for example, $\lambda>$ 1 for population growth, or $\beta<0$ for a slope in a regression model), we report the posterior probability $(\operatorname{Pr}[\mathrm{x}]=$ probability that $\mathrm{x}$ is true $)$ that the parameter is beyond that threshold.

\section{Results}

\section{Capture Summary}

We made 2,995 total captures of 1,011 individual snakes from 2014-17. In 2016, we captured 540 individual snakes a total of 1,567 times, whereas in 2017, we captured 423 snakes a total of 999 times. Trapping effort was highest in 2016 and lowest in 2014, and we captured fewer snakes in 2014 and 2015 when trapping effort was relatively low. Trapping effort in 2017 was lower than 2016 because delayed flooding of canals in 2017 meant trapping began later at most sites compared to 2016 (table 1).

The frequency of year-over-year recaptures (a snake captured in more than 1 year) varied among sites (table 2). Of the 1,011 snakes captured at all sites, 816 were captured in 1 year, 178 were captured in 2 years, 15 were captured in 3 years, and 2 individuals were captured all 4 years. The recapture rate was lowest at site 4, which was trapped for 3 years (2015-17). At site 4, 94.6 percent of snakes were only captured in 1 year: the remaining 5.4 percent of snakes were captured in 2 years. Year-over-year recaptures were more frequent at site 5 (31.0 percent of snakes captured in more than 1 year) and site 6 (29.8 percent of snakes captured in more than 1 year). At the remaining sites, 15.8-22.7 percent of snakes were captured in more than 1 year (table 2). The recapture rate of snakes in the year following their initial capture was higher in 2016 and 2017 than 2015. Of 124 snakes first captured in 2014, 18 were recaptured in 2015 (14.5 percent). Of 120 snakes first captured in 2015, 42 were recaptured in 2016 (35.0 percent). Of 480 snakes first captured in 2016, 118 were recaptured in 2017 (24.6 percent). Two snakes moved from site 3 to site 5 between 2016 and 2017. 
Table 2. Frequency of year-over-year recaptures of giant gartersnakes by site in the Sacramento Valley, California, from 2014-17.

[Note that sites 4, 6, and 7 were only sampled in 3 years; all other sites were sampled in 4 years. -- indicates that captures in 4 years were not possible at these sites]

\begin{tabular}{|c|c|c|c|c|c|c|}
\hline \multirow[b]{2}{*}{ Site } & \multirow[b]{2}{*}{ Years sampled } & \multicolumn{4}{|c|}{ \# of Individuals captured in } & \multirow[b]{2}{*}{ Total } \\
\hline & & 1 year & 2 years & 3 years & 4 years & \\
\hline 1 & 4 & 127 & 32 & 4 & 1 & 164 \\
\hline 2 & 4 & 123 & 23 & 0 & 0 & 146 \\
\hline 3 & 4 & 122 & 25 & 2 & 0 & 149 \\
\hline 4 & 3 & 106 & 6 & 0 & -- & 112 \\
\hline 5 & 4 & 29 & 9 & 4 & 0 & 42 \\
\hline 6 & 3 & 80 & 29 & 5 & -- & 114 \\
\hline 7 & 3 & 34 & 10 & 0 & -- & 44 \\
\hline 8 & 4 & 195 & 44 & 0 & 1 & 240 \\
\hline All & & 816 & 178 & 15 & 2 & 1,011 \\
\hline
\end{tabular}

\section{Reproductive Exams}

We examined the reproductive status of 21 females in 2014, 37 females in 2015, 37 females in 2016, and 80 females in 2017 (table 3). Because the reproductive data for 2014-16 have been summarized previously (Rose and others, 2018a,c), we focus here on results from 2017, the first full year in which reproductive data were collected for the current study. In 2017, we examined females from May 15 to July 11, and no females appeared to have given birth prior to examination. In 2017,51 of 80 females were gravid. The smallest gravid female was an individual of $500 \mathrm{~mm}$ SVL collected at site 4 in 2017; the smallest female examined was 435 $\mathrm{mm}$ SVL and was not gravid. Over 70 percent of examined females were gravid at sites 1, 2, 4, and 8 in 2017, whereas fewer than half of examined females were gravid at site 2 (table 3 ). The size of examined females varied among sites. On average, examined females were smallest at site 4 and largest at site 5 (table 3 ).

Both the probability a female was gravid $\left(\beta g_{S V L}=1.604,95\right.$-percent CRI $\left.=0.832-2.479\right)$ and the estimated litter size $\left(\beta \mathrm{f}_{\mathrm{SVL}}=0.299,0.203-0.394\right)$ were positively related to a female's SVL (fig. 2). Based on our models, a female of average SVL $(718 \mathrm{~mm})$ would have a 68 percent chance of being gravid and would be expected to produce a litter of 16 offspring on average. There was no support for linear $\left(\beta_{\text {date }}=0.188,-.400-0.791\right)$ or quadratic $\left(\beta_{\text {date } 2}=-0.084\right.$, $-0.803-0.670$ ) effects of date of examination on reproductive status because both parameters substantially overlapped zero. After accounting for female SVL, the differences among sites in the probability a female was gravid and litter size were minimal (fig. 3). 
Table 3. Summary of reproductive data of giant gartersnakes collected from the Sacramento Valley, California, 2014-17, by site and year.

[Litter size is a count of eggs and embryos from X-rays. 2014 and 2015 include data from radio-telemetry snakes examined as part of a previous study (Reyes and others, 2017), 2016 includes females examined for the current study and radio-telemetry females examined by Reyes and others (2017), and 2017 includes data from the current study only. SD = standard deviation]

\begin{tabular}{|c|c|c|c|c|c|c|c|c|c|}
\hline \multirow[b]{2}{*}{ Year } & \multirow[b]{2}{*}{ Site } & \multirow[b]{2}{*}{$\mathrm{N}$} & \multirow[b]{2}{*}{ Gravid } & \multicolumn{4}{|c|}{ Litter size } & \multicolumn{2}{|c|}{ Female SVL } \\
\hline & & & & Mean & SD & Min & Max & Mean & SD \\
\hline 2014 & 1 & 5 & 0 & -- & -- & -- & -- & 763.4 & 81.4 \\
\hline 2014 & 2 & 2 & 1 & 15.0 & -- & -- & -- & 767.5 & 24.7 \\
\hline 2014 & 3 & 5 & 3 & 15.3 & 3.2 & 13 & 19 & 735.0 & 70.1 \\
\hline 2014 & 4 & 0 & -- & -- & -- & -- & -- & -- & -- \\
\hline 2014 & 5 & 4 & 1 & 17.0 & -- & -- & -- & 824.8 & 37.3 \\
\hline 2014 & 6 & 0 & -- & -- & -- & -- & -- & -- & -- \\
\hline 2014 & 7 & 0 & -- & -- & -- & -- & -- & -- & -- \\
\hline 2014 & 8 & 5 & 5 & 14.0 & 1.6 & 12 & 16 & 712.8 & 40.6 \\
\hline 2015 & 1 & 6 & 4 & 15.3 & 1.7 & 13 & 17 & 742.9 & 62.7 \\
\hline 2015 & 2 & 2 & 0 & -- & -- & -- & -- & 844.0 & 22.6 \\
\hline 2015 & 3 & 5 & 1 & 13.0 & -- & -- & -- & 751.5 & 64.7 \\
\hline 2015 & 4 & 5 & 3 & 16.3 & 3.8 & 12 & 19 & 786.8 & 24.2 \\
\hline 2015 & 5 & 5 & 1 & 15.0 & -- & -- & -- & 834.0 & 50.4 \\
\hline 2015 & 6 & 4 & 2 & 11.0 & 1.4 & 10 & 12 & 736.0 & 49.4 \\
\hline 2015 & 7 & 5 & 4 & 23.8 & 6.9 & 18 & 33 & 872.6 & 129.6 \\
\hline 2015 & 8 & 5 & 2 & 16.5 & 0.7 & 17 & 16 & 810.4 & 47.0 \\
\hline 2016 & 1 & 5 & 3 & 17.0 & 6.9 & 9 & 21 & 759.9 & 142.6 \\
\hline 2016 & 2 & 3 & 1 & 12.0 & -- & -- & -- & 822.7 & 74.3 \\
\hline 2016 & 3 & 4 & 3 & 16.3 & 5.5 & 11 & 22 & 749.8 & 77.0 \\
\hline 2016 & 4 & 4 & 3 & 14.0 & 3.5 & 10 & 16 & 738.5 & 159.2 \\
\hline 2016 & 5 & 5 & 1 & 21.0 & -- & -- & -- & 845.1 & 20.2 \\
\hline 2016 & 6 & 3 & 3 & 15.3 & 4.9 & 12 & 21 & 771.3 & 68.6 \\
\hline 2016 & 7 & 7 & 2 & 20.0 & 2.8 & 18 & 22 & 914.7 & 106.0 \\
\hline 2016 & 8 & 6 & 5 & 14.4 & 5.4 & 18 & 19 & 763.8 & 89.2 \\
\hline 2017 & 1 & 7 & 5 & 18.8 & 2.6 & 16 & 23 & 708.0 & 144.1 \\
\hline 2017 & 2 & 12 & 5 & 19.3 & 4.3 & 13 & 22 & 693.2 & 127.5 \\
\hline 2017 & 3 & 14 & 10 & 21.5 & 6.5 & 13 & 34 & 757.9 & 134.5 \\
\hline 2017 & 4 & 11 & 8 & 11.6 & 4.3 & 7 & 18 & 589.2 & 89.4 \\
\hline 2017 & 5 & 8 & 5 & 18.6 & 3.5 & 15 & 24 & 789.9 & 85.1 \\
\hline 2017 & 6 & 10 & 6 & 22.3 & 6.4 & 15 & 34 & 731.6 & 69.1 \\
\hline 2017 & 7 & 4 & 2 & 23.0 & 2.8 & 21 & 25 & 730.3 & 113.7 \\
\hline 2017 & 8 & 14 & 10 & 17.2 & 4.8 & 10 & 24 & 749.8 & 96.4 \\
\hline
\end{tabular}



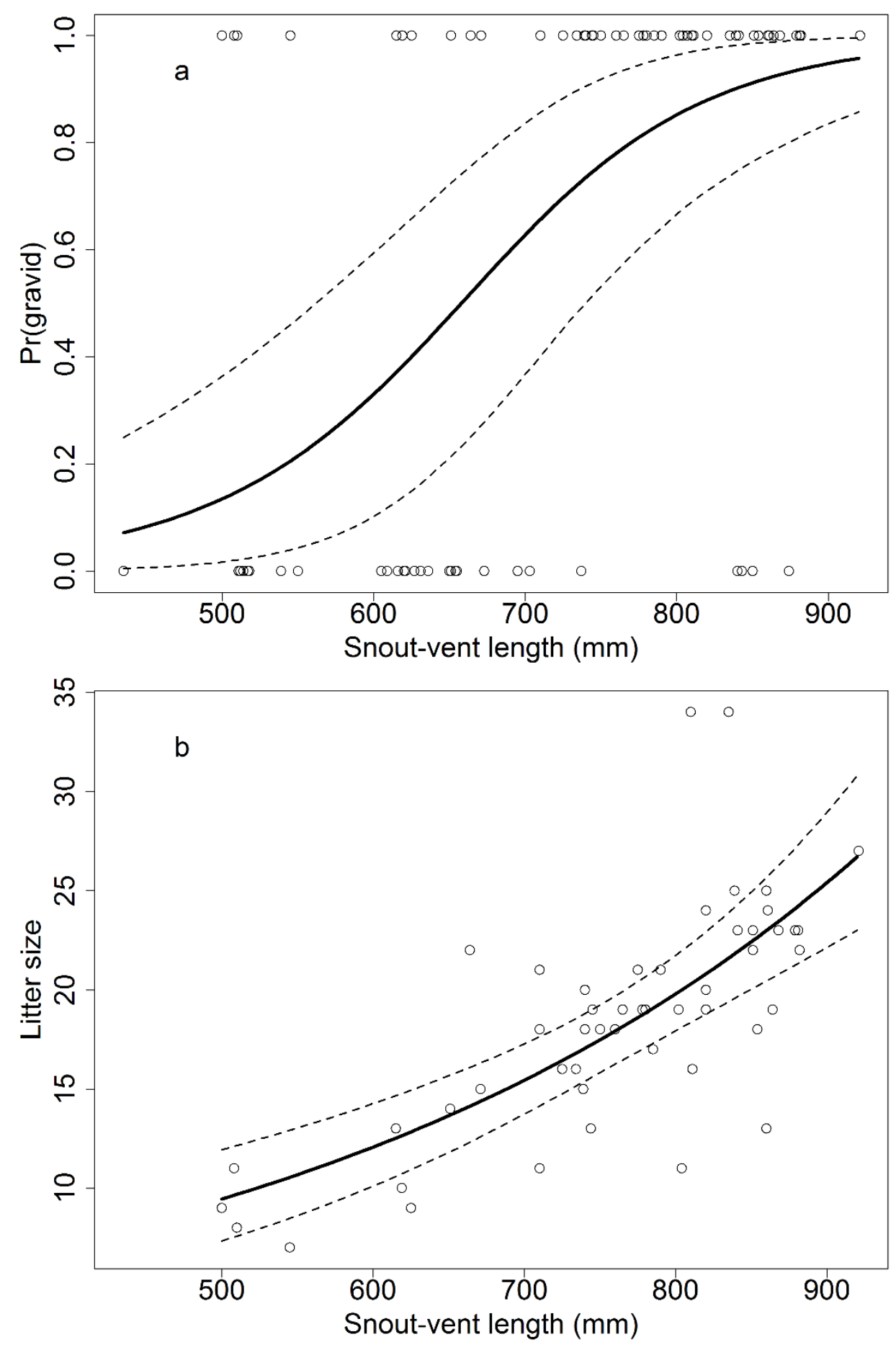

Figure 2. The probability a female giant gartersnake was gravid (a) and the observed litter size of gravid females (b) versus female snout-vent length (SVL). Circles represent observed data from X-rays of female snakes collected from the Sacramento Valley, California, in 2017. In panel a, a value of 1 on the $y$-axis indicates a female was gravid, and a value of 0 indicates a female was not gravid. The solid lines are the mean predicted relationship, and dashed lines represent 95-percent credible intervals around this mean relationship. $\operatorname{Pr}($ gravid $)=$ probability a female was gravid. 

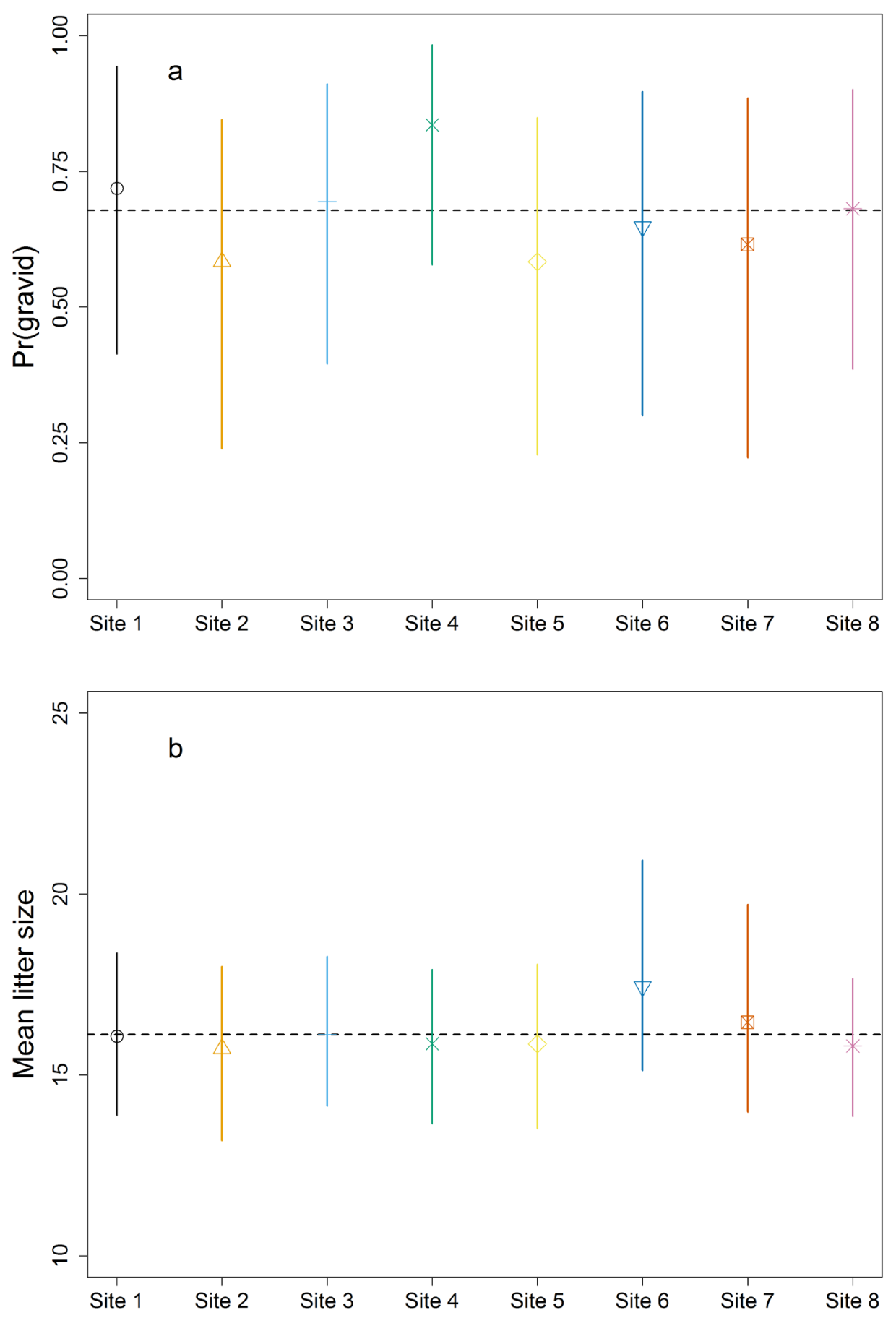

Figure 3. Site-specific estimates for the mean probability a female giant gartersnake was gravid (a) and mean litter size (b) for each site in the Sacramento Valley, California. Data are from females with snoutvent length near or $>500$ millimeters that received $X$-rays in 2017 . The dashed line represents the average parameter estimate for all sites. $\operatorname{Pr}($ gravid $)=$ probability a female was gravid. 


\section{Abundance and Population Growth Estimates}

The highest abundance of giant gartersnakes was found at site 8 each year (table 4; fig. 4). In contrast, the estimated abundance of giant gartersnakes was low each year at sites 5 and 7, which had the fewest captures each year. The mean abundance estimates were higher in 2016 and 2017 than in 2014 and 2015 for sites 1-4 and 6 (table 4; fig. 4). This apparent increase in abundance in the 2 most recent years could be an artifact of the increase in sampling effort and sampled area in 2016 and 2017 compared to 2014 and 2015 (table 1). The abundance estimates for site 8 and site 2 in 2014 and 2015 have wide credible intervals because of the limited sampling at both sites in those years (fig. 4).

Although the mean estimates of abundance at a site appeared to change from year to year, the estimates of $\lambda$ illustrate the uncertainty in the population growth rate. The 95 -percent credible interval for all estimates of $\lambda$ overlapped one (fig. 5). A value of $\lambda=1$ represents a stable population. There was great uncertainty in the estimates of $\lambda$ for 2014-15, which reflects the lower sampling effort in these years and consequently the high uncertainty in estimates of abundance (fig. 5). The estimates of $\lambda$ for 2016-17 were more precise, and there was some evidence that the abundance of giant gartersnakes declined during this time period at site 2 $(\operatorname{Pr}[\hat{\lambda}<1]=0.95)$, site $4(\operatorname{Pr}[\hat{\lambda}<1]=0.95)$, and site $7(\operatorname{Pr}[\hat{\lambda}<1]=0.94)$, although the credible intervals overlapped one slightly (fig. 5). It is worth noting that our sampling effort was lower in 2017 than 2016 at each of these three sites (table 1). There also was some evidence that the population grew from $2016-17$ at site $5(\operatorname{Pr}[\hat{\lambda}>1]=0.93)$, although the credible interval for $\hat{\lambda}$ again overlapped one slightly (fig. 5).

The mean estimated daily capture probability $(p)$ was higher in 2016 than 2014 and 2015 at most sites, but in general, variation between years within a site was low (table 5; fig. 6). In contrast, the probability of an individual being captured at least once (that is, annual capture probability, $\left.p^{*}\right)$ in a year increased from 2014-16 at most sites, reflecting the increased sampling effort at each site in 2016 (tables 1 and 6; fig. 7). The higher sampling effort in 2016 resulted in more precise estimates of abundance in that year compared to previous years (fig. 4). Although sampling effort declined slightly in 2017 compared to 2016, the daily and annual capture probabilities in 2017 were similar to those in 2016 (tables 5 and 6; figs. 6 and 7). 
Table 4. Giant gartersnake abundance estimates $(\widehat{N})$ by site and year for eight study sites in the Sacramento Valley, California, 2014-17.

\begin{tabular}{|c|c|c|c|c|c|}
\hline \multirow[b]{2}{*}{ Site } & \multirow[b]{2}{*}{ Year } & \multicolumn{4}{|c|}{$\widehat{N}$} \\
\hline & & Mean & SD & $2.50 \%$ & $97.50 \%$ \\
\hline 1 & 2014 & 78 & 12 & 58 & 106 \\
\hline 1 & 2015 & 53 & 8 & 40 & 72 \\
\hline 1 & 2016 & 100 & 8 & 87 & 117 \\
\hline 1 & 2017 & 101 & 9 & 85 & 121 \\
\hline 2 & 2014 & 37 & 13 & 21 & 69 \\
\hline 2 & 2015 & 29 & 13 & 13 & 61 \\
\hline 2 & 2016 & 123 & 10 & 106 & 145 \\
\hline 2 & 2017 & 99 & 12 & 80 & 125 \\
\hline 3 & 2014 & 72 & 19 & 45 & 117 \\
\hline 3 & 2015 & 61 & 14 & 41 & 93 \\
\hline 3 & 2016 & 109 & 10 & 92 & 132 \\
\hline 3 & 2017 & 106 & 14 & 83 & 137 \\
\hline 4 & 2015 & 47 & 12 & 29 & 76 \\
\hline 4 & 2016 & 86 & 10 & 70 & 109 \\
\hline 4 & 2017 & 66 & 8 & 52 & 85 \\
\hline 5 & 2014 & 32 & 12 & 15 & 61 \\
\hline 5 & 2015 & 30 & 8 & 17 & 48 \\
\hline 5 & 2016 & 29 & 5 & 21 & 41 \\
\hline 5 & 2017 & 39 & 7 & 28 & 55 \\
\hline 6 & 2015 & 48 & 6 & 37 & 62 \\
\hline 6 & 2016 & 106 & 7 & 93 & 122 \\
\hline 6 & 2017 & 90 & 14 & 66 & 121 \\
\hline 7 & 2015 & 26 & 6 & 17 & 42 \\
\hline 7 & 2016 & 43 & 7 & 32 & 58 \\
\hline 7 & 2017 & 28 & 8 & 16 & 47 \\
\hline 8 & 2014 & 115 & 31 & 68 & 189 \\
\hline 8 & 2015 & 101 & 44 & 48 & 217 \\
\hline 8 & 2016 & 171 & 11 & 152 & 194 \\
\hline 8 & 2017 & 188 & 14 & 164 & 218 \\
\hline
\end{tabular}




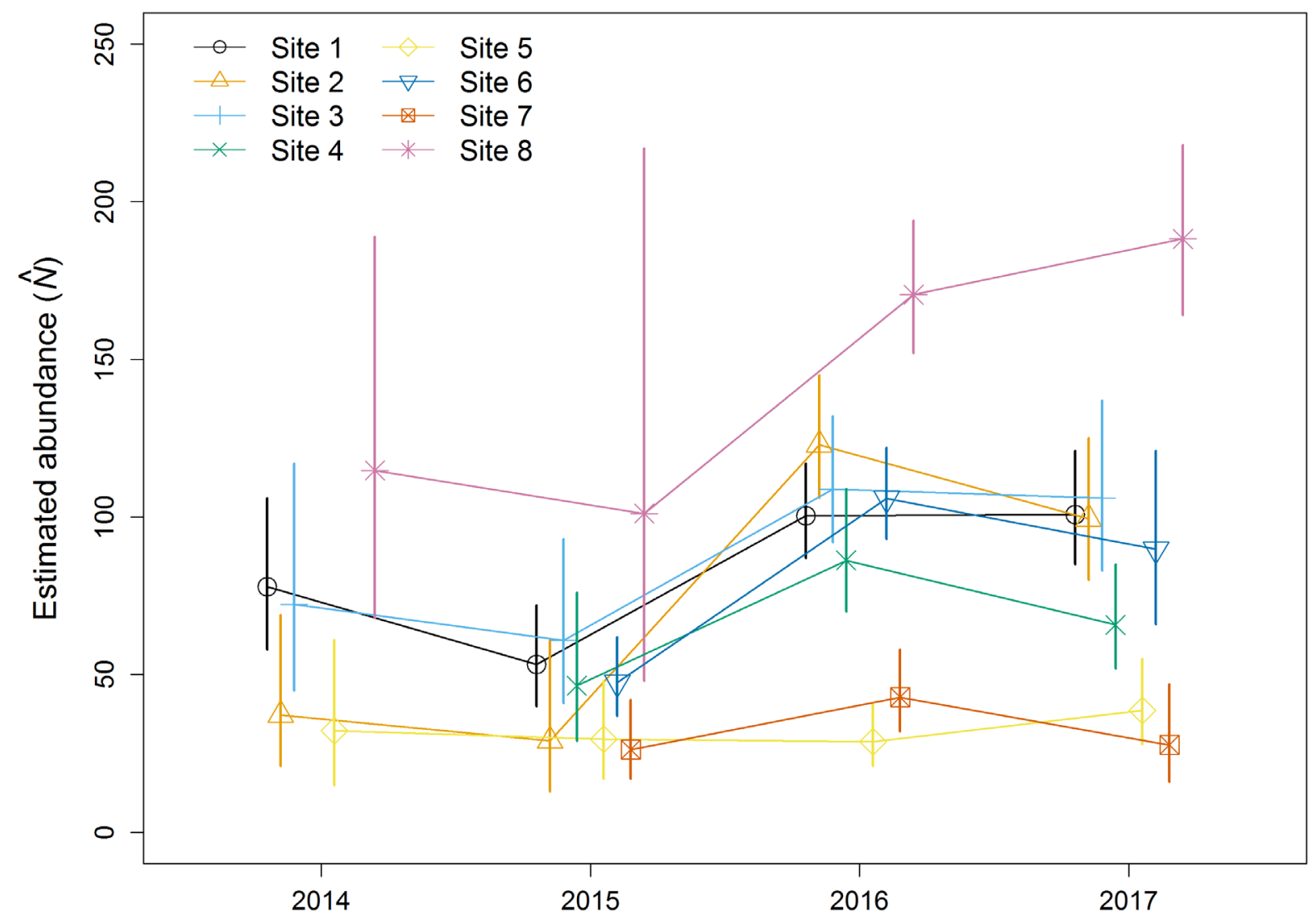

Figure 4. Estimated abundance $(\widehat{N})$ of giant gartersnakes at each site in the Sacramento Valley, California, from 2014-17. 


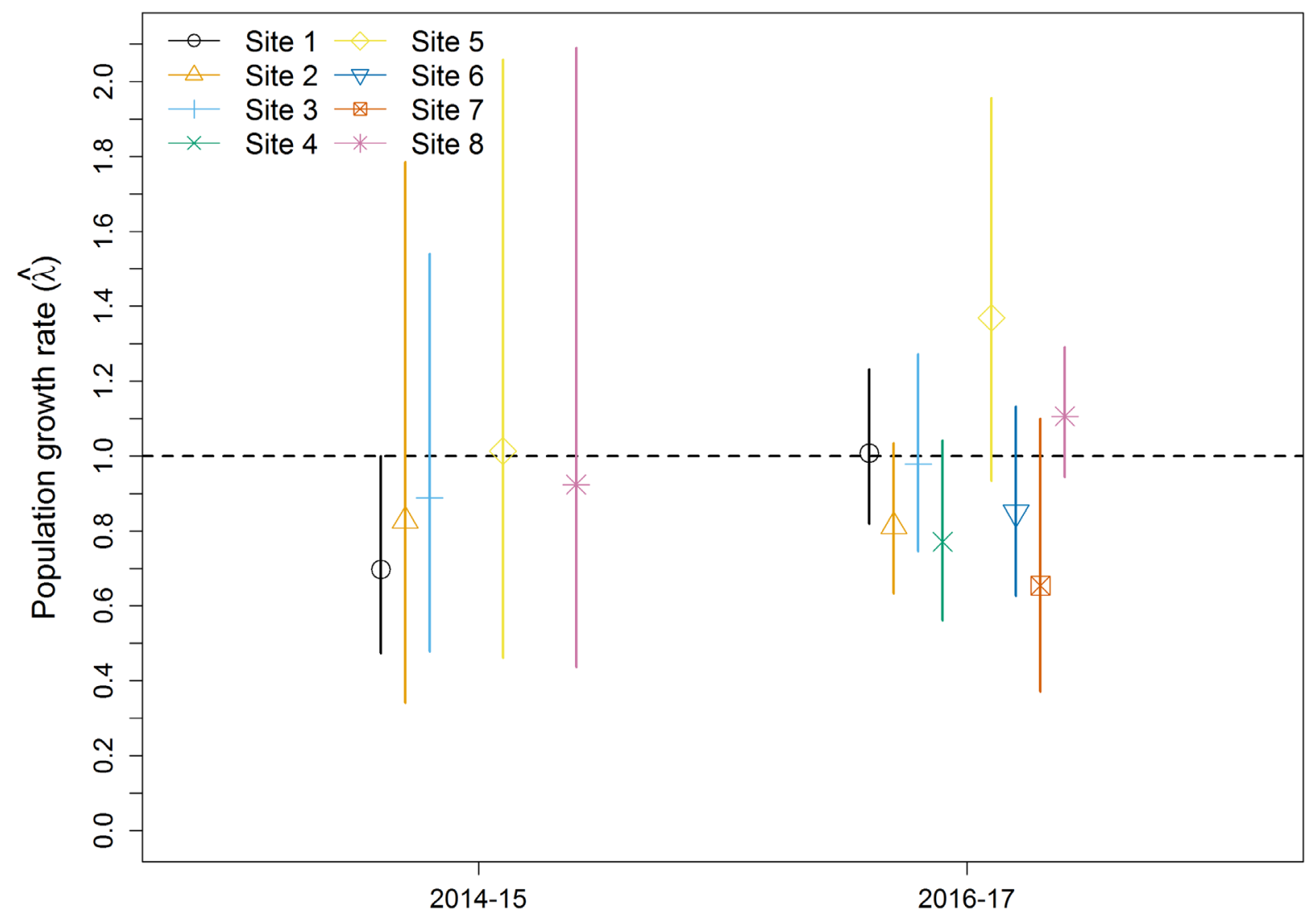

Figure 5. Estimated population growth rate $(\hat{\lambda})$ of giant gartersnakes for 2014-15 and 2016-17 from eight sites in the Sacramento Valley, California. 
Table 5. Giant gartersnake daily capture probability estimates $(\hat{p})$ by site and year for eight study sites in the Sacramento Valley, California, 2014-17.

\begin{tabular}{|c|c|c|c|c|c|}
\hline \multirow[b]{2}{*}{ Site } & \multirow[b]{2}{*}{ Year } & \multicolumn{4}{|c|}{$\widehat{\boldsymbol{p}}$} \\
\hline & & Mean & SD & $2.50 \%$ & $97.50 \%$ \\
\hline 1 & 2014 & 0.032 & 0.008 & 0.020 & 0.049 \\
\hline 1 & 2015 & 0.009 & 0.002 & 0.005 & 0.014 \\
\hline 1 & 2016 & 0.018 & 0.003 & 0.013 & 0.024 \\
\hline 1 & 2017 & 0.017 & 0.003 & 0.012 & 0.023 \\
\hline 2 & 2014 & 0.005 & 0.002 & 0.002 & 0.009 \\
\hline 2 & 2015 & 0.007 & 0.003 & 0.002 & 0.014 \\
\hline 2 & 2016 & 0.017 & 0.003 & 0.013 & 0.023 \\
\hline 2 & 2017 & 0.012 & 0.002 & 0.008 & 0.017 \\
\hline 3 & 2014 & 0.007 & 0.002 & 0.004 & 0.013 \\
\hline 3 & 2015 & 0.004 & 0.001 & 0.002 & 0.007 \\
\hline 3 & 2016 & 0.015 & 0.003 & 0.011 & 0.021 \\
\hline 3 & 2017 & 0.010 & 0.002 & 0.006 & 0.014 \\
\hline 4 & 2015 & 0.012 & 0.004 & 0.006 & 0.020 \\
\hline 4 & 2016 & 0.015 & 0.003 & 0.010 & 0.022 \\
\hline 4 & 2017 & 0.027 & 0.006 & 0.017 & 0.040 \\
\hline 5 & 2014 & 0.010 & 0.004 & 0.004 & 0.021 \\
\hline 5 & 2015 & 0.005 & 0.002 & 0.002 & 0.009 \\
\hline 5 & 2016 & 0.011 & 0.003 & 0.006 & 0.018 \\
\hline 5 & 2017 & 0.012 & 0.003 & 0.007 & 0.020 \\
\hline 6 & 2015 & 0.013 & 0.003 & 0.008 & 0.019 \\
\hline 6 & 2016 & 0.019 & 0.003 & 0.014 & 0.024 \\
\hline 6 & 2017 & 0.009 & 0.002 & 0.006 & 0.014 \\
\hline 7 & 2015 & 0.008 & 0.003 & 0.004 & 0.015 \\
\hline 7 & 2016 & 0.012 & 0.003 & 0.007 & 0.020 \\
\hline 7 & 2017 & 0.013 & 0.005 & 0.006 & 0.025 \\
\hline 8 & 2014 & 0.013 & 0.004 & 0.006 & 0.022 \\
\hline 8 & 2015 & 0.012 & 0.006 & 0.004 & 0.026 \\
\hline 8 & 2016 & 0.019 & 0.002 & 0.015 & 0.024 \\
\hline 8 & 2017 & 0.014 & 0.002 & 0.011 & 0.018 \\
\hline
\end{tabular}


Table 6. Giant gartersnake annual capture probability estimates $\left(\hat{p}^{*}\right)$ by site and year for eight study sites in the Sacramento Valley, California, 2014-17.

\begin{tabular}{|c|c|c|c|c|c|}
\hline \multirow[b]{2}{*}{ Site } & \multirow[b]{2}{*}{ Year } & \multicolumn{4}{|c|}{$\widehat{\boldsymbol{p}}^{*}$} \\
\hline & & Mean & SD & $2.50 \%$ & $97.50 \%$ \\
\hline 1 & 2014 & 0.52 & 0.08 & 0.37 & 0.69 \\
\hline 1 & 2015 & 0.49 & 0.08 & 0.33 & 0.65 \\
\hline 1 & 2016 & 0.77 & 0.05 & 0.67 & 0.86 \\
\hline 1 & 2017 & 0.72 & 0.06 & 0.60 & 0.83 \\
\hline 2 & 2014 & 0.40 & 0.12 & 0.18 & 0.65 \\
\hline 2 & 2015 & 0.23 & 0.09 & 0.09 & 0.42 \\
\hline 2 & 2016 & 0.76 & 0.05 & 0.65 & 0.85 \\
\hline 2 & 2017 & 0.60 & 0.07 & 0.47 & 0.73 \\
\hline 3 & 2014 & 0.35 & 0.09 & 0.19 & 0.53 \\
\hline 3 & 2015 & 0.39 & 0.09 & 0.23 & 0.57 \\
\hline 3 & 2016 & 0.71 & 0.06 & 0.59 & 0.82 \\
\hline 3 & 2017 & 0.49 & 0.07 & 0.36 & 0.63 \\
\hline 4 & 2015 & 0.42 & 0.10 & 0.24 & 0.62 \\
\hline 4 & 2016 & 0.65 & 0.07 & 0.51 & 0.79 \\
\hline 4 & 2017 & 0.71 & 0.08 & 0.55 & 0.85 \\
\hline 5 & 2014 & 0.30 & 0.11 & 0.13 & 0.54 \\
\hline 5 & 2015 & 0.41 & 0.12 & 0.21 & 0.66 \\
\hline 5 & 2016 & 0.62 & 0.10 & 0.42 & 0.81 \\
\hline 5 & 2017 & 0.53 & 0.09 & 0.35 & 0.71 \\
\hline 6 & 2015 & 0.69 & 0.07 & 0.53 & 0.83 \\
\hline 6 & 2016 & 0.81 & 0.05 & 0.71 & 0.89 \\
\hline 6 & 2017 & 0.44 & 0.07 & 0.31 & 0.58 \\
\hline 7 & 2015 & 0.59 & 0.12 & 0.35 & 0.82 \\
\hline 7 & 2016 & 0.64 & 0.09 & 0.45 & 0.81 \\
\hline 7 & 2017 & 0.47 & 0.12 & 0.26 & 0.71 \\
\hline 8 & 2014 & 0.23 & 0.06 & 0.13 & 0.37 \\
\hline 8 & 2015 & 0.05 & 0.02 & 0.02 & 0.10 \\
\hline 8 & 2016 & 0.81 & 0.04 & 0.72 & 0.88 \\
\hline 8 & 2017 & 0.68 & 0.05 & 0.58 & 0.77 \\
\hline
\end{tabular}




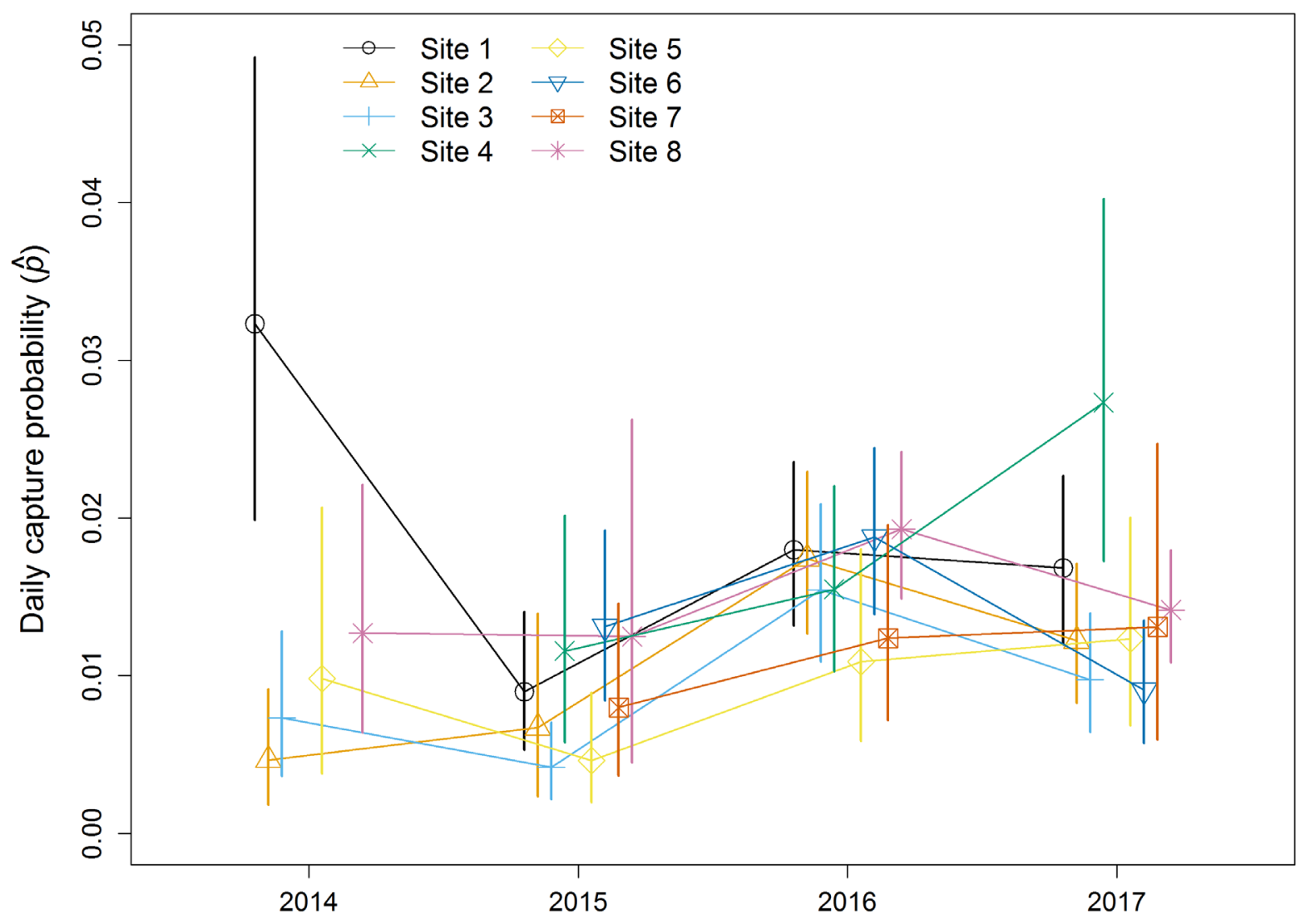

Figure 6. Estimated daily capture probability $(\hat{p})$ of giant gartersnakes from 2014-17 for eight sites in the Sacramento Valley, California. 


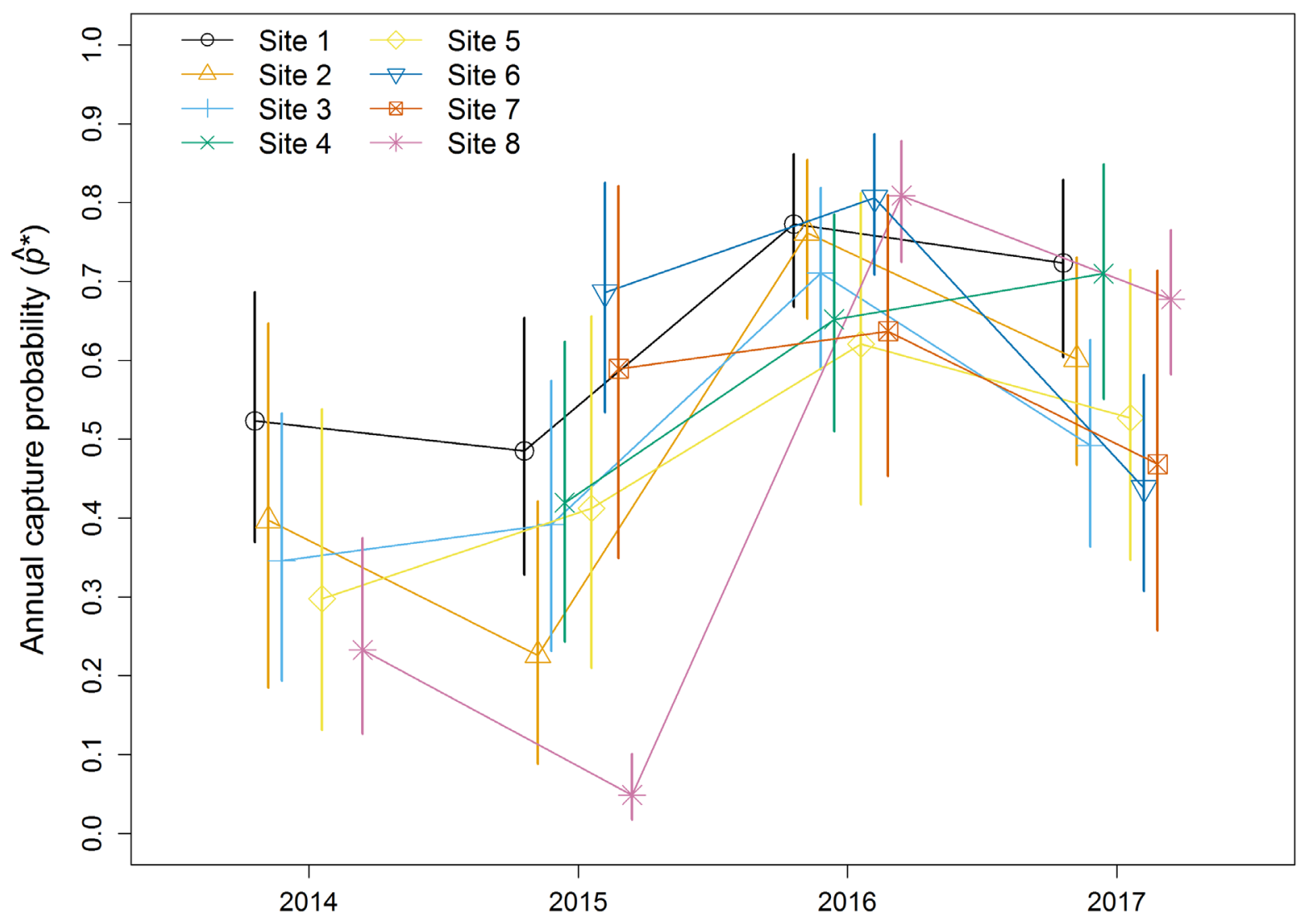

Figure 7. Estimated annual capture probability $\left(\hat{p}^{*}\right)$ of giant gartersnakes from 2014-17 for eight sites in the Sacramento Valley, California. 


\section{Annual Apparent Survival Estimates}

The mean estimate of annual apparent survival probability (for snakes of all sizes) averaged over all 8 study sites was 0.40 (95-percent CRI $=0.26-0.54)$. Annual apparent survival varied among sites, but estimates did not vary much over time within a site. Survival was lowest at site 4, where the mean estimate of annual apparent survival probability was less than 0.2 for 2015-16 and 2016-17. Survival was highest at site 5, where the mean estimate of annual apparent survival probability was greater than 0.5 each year (table 7 ; fig. 8). There were no clear trends in survival over time during this short time series. Within a site, the credible intervals for survival overlapped substantially between years (fig. 8).

Table 7. Giant gartersnake annual apparent survival estimates $(\widehat{\phi})$ by site and year for eight study sites in the Sacramento Valley, California, 2014-17.

\begin{tabular}{|c|c|c|c|c|c|}
\hline \multirow[b]{2}{*}{ Site } & \multirow[b]{2}{*}{ Year } & \multicolumn{4}{|c|}{$\widehat{\phi}$} \\
\hline & & Mean & SD & $2.50 \%$ & $97.50 \%$ \\
\hline 1 & 2014 & 0.46 & 0.08 & 0.33 & 0.63 \\
\hline 1 & 2015 & 0.42 & 0.07 & 0.28 & 0.55 \\
\hline 1 & 2016 & 0.40 & 0.06 & 0.28 & 0.51 \\
\hline 2 & 2014 & 0.32 & 0.09 & 0.17 & 0.52 \\
\hline 2 & 2015 & 0.34 & 0.09 & 0.19 & 0.56 \\
\hline 2 & 2016 & 0.32 & 0.06 & 0.21 & 0.43 \\
\hline 3 & 2014 & 0.37 & 0.08 & 0.22 & 0.54 \\
\hline 3 & 2015 & 0.35 & 0.07 & 0.21 & 0.49 \\
\hline 3 & 2016 & 0.39 & 0.07 & 0.27 & 0.54 \\
\hline 4 & 2015 & 0.19 & 0.08 & 0.07 & 0.38 \\
\hline 4 & 2016 & 0.14 & 0.05 & 0.06 & 0.26 \\
\hline 5 & 2014 & 0.52 & 0.13 & 0.25 & 0.75 \\
\hline 5 & 2015 & 0.59 & 0.10 & 0.38 & 0.79 \\
\hline 5 & 2016 & 0.63 & 0.10 & 0.43 & 0.83 \\
\hline 6 & 2015 & 0.56 & 0.08 & 0.41 & 0.72 \\
\hline 6 & 2016 & 0.51 & 0.08 & 0.35 & 0.68 \\
\hline 7 & 2015 & 0.39 & 0.11 & 0.21 & 0.62 \\
\hline 7 & 2016 & 0.35 & 0.10 & 0.18 & 0.57 \\
\hline 8 & 2014 & 0.46 & 0.08 & 0.31 & 0.65 \\
\hline 8 & 2015 & 0.47 & 0.09 & 0.33 & 0.67 \\
\hline 8 & 2016 & 0.42 & 0.05 & 0.32 & 0.52 \\
\hline
\end{tabular}




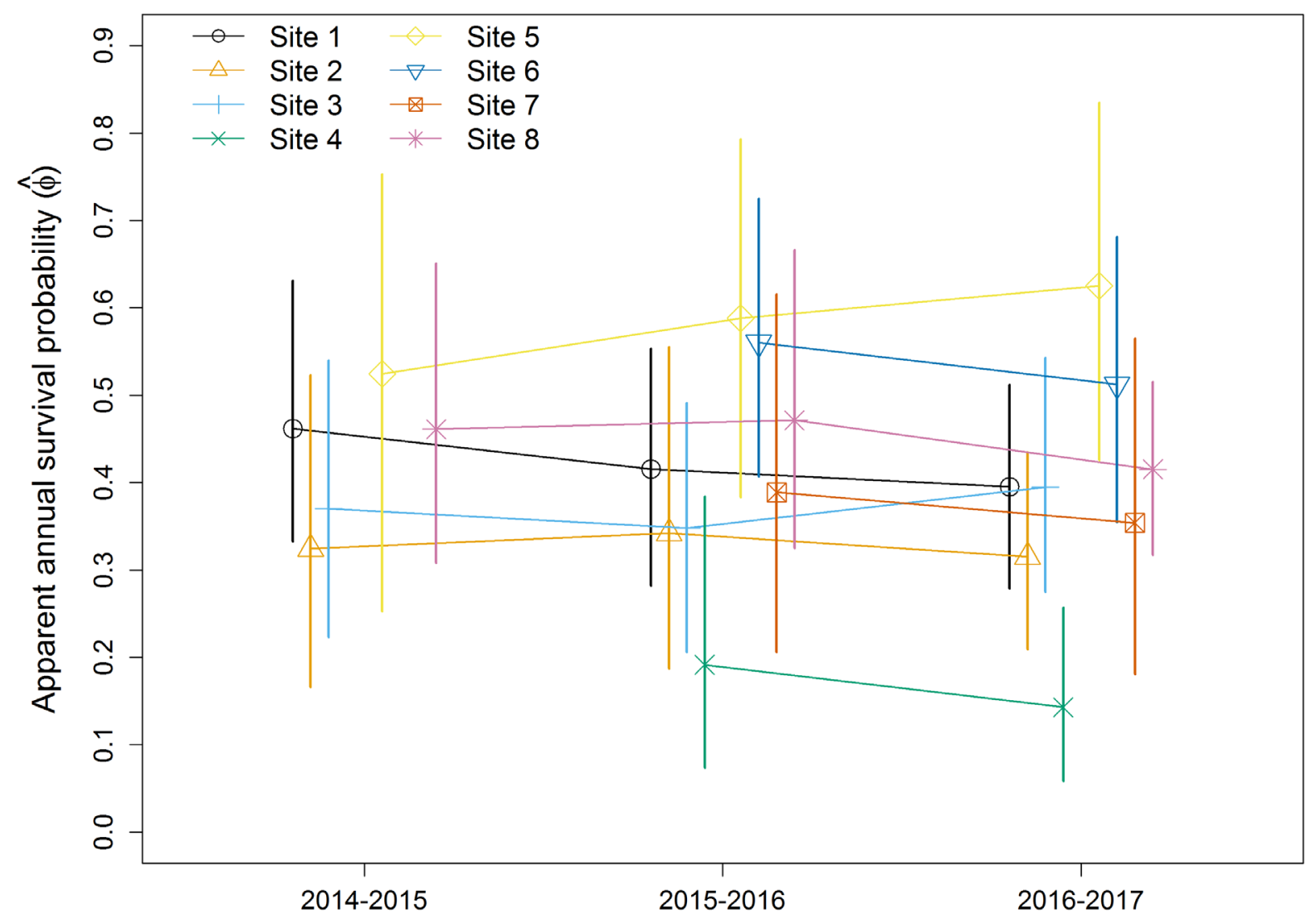

Figure 8. Estimated annual apparent survival $(\widehat{\phi})$ of giant gartersnakes from 2014-17 for eight sites in the Sacramento Valley, California.

\section{Rice Growing Trends}

For most sites in most years, the proportional area of rice growing within a $1-\mathrm{km}$ buffer varied between 60 and 90 percent (range 29-88 percent; interquartile range $=62-80$ percent), although this proportion was below 50 percent for some sites in some years. The amount of rice grown varied over time at each site. In general, the area of rice growing increased from 2014 to 2016 and then declined in 2017, but some sites did not follow the same trends, and the magnitude of the changes between years varied among sites (table 8; fig. 9). For example, the area of rice growing more than doubled from 2014-15, then declined sharply from 2015 to 2017 at site 8 , while the amount of rice growing remained fairly consistent over the same period at sites $2-4$. The amount of rice growing fluctuated the most at site 6 , with the proportion of rice alternating from greater than 80 percent in 2014 and 2016 to less than 55 percent in 2015 and 2017. 
Table 8. Extent of rice growing and other land cover types within a 1-km buffer of eight study sites in the Sacramento Valley, California, from 2014-17.

[Proportions are based on classified Landsat 8 data. For 2017, no data are available yet on fallow fields, non-rice crops, and other land covers]

\begin{tabular}{|c|c|c|c|c|c|}
\hline Site & Year & Rice & Fallow & Non-rice crop & Other \\
\hline 1 & 2014 & 0.62 & 0.32 & 0.01 & 0.05 \\
\hline 1 & 2015 & 0.62 & 0.33 & 0.01 & 0.04 \\
\hline 1 & 2016 & 0.86 & 0.09 & 0.00 & 0.05 \\
\hline 1 & 2017 & 0.76 & -- & -- & -- \\
\hline 2 & 2014 & 0.71 & 0.15 & 0.02 & 0.12 \\
\hline 2 & 2015 & 0.7 & 0.19 & 0.01 & 0.1 \\
\hline 2 & 2016 & 0.76 & 0.13 & 0.02 & 0.10 \\
\hline 2 & 2017 & 0.65 & -- & -- & -- \\
\hline 3 & 2014 & 0.66 & 0.28 & 0.01 & 0.04 \\
\hline 3 & 2015 & 0.74 & 0.2 & 0.01 & 0.05 \\
\hline 3 & 2016 & 0.84 & 0.10 & 0.01 & 0.05 \\
\hline 3 & 2017 & 0.75 & -- & -- & -- \\
\hline 4 & 2014 & 0.76 & 0.22 & 0.01 & 0.01 \\
\hline 4 & 2015 & 0.83 & 0.14 & 0.02 & 0.01 \\
\hline 4 & 2016 & 0.87 & 0.12 & 0.00 & 0.01 \\
\hline 4 & 2017 & 0.69 & -- & -- & -- \\
\hline 5 & 2014 & 0.61 & 0.29 & 0.06 & 0.03 \\
\hline 5 & 2015 & 0.77 & 0.13 & 0.07 & 0.03 \\
\hline 5 & 2016 & 0.75 & 0.12 & 0.09 & 0.04 \\
\hline 5 & 2017 & 0.62 & -- & -- & -- \\
\hline 6 & 2014 & 0.85 & 0.11 & 0.01 & 0.03 \\
\hline 6 & 2015 & 0.54 & 0.41 & 0.01 & 0.04 \\
\hline 6 & 2016 & 0.88 & 0.09 & 0.00 & 0.04 \\
\hline 6 & 2017 & 0.35 & -- & -- & -- \\
\hline 7 & 2014 & 0.79 & 0.11 & 0.09 & 0.01 \\
\hline 7 & 2015 & 0.82 & 0.16 & 0.01 & 0.01 \\
\hline 7 & 2016 & 0.87 & 0.08 & 0.04 & 0.01 \\
\hline 7 & 2017 & 0.63 & -- & -- & -- \\
\hline 8 & 2014 & 0.30 & 0.38 & 0.27 & 0.05 \\
\hline 8 & 2015 & 0.63 & 0.13 & 0.19 & 0.05 \\
\hline 8 & 2016 & 0.50 & 0.13 & 0.32 & 0.05 \\
\hline 8 & 2017 & 0.29 & -- & -- & -- \\
\hline
\end{tabular}




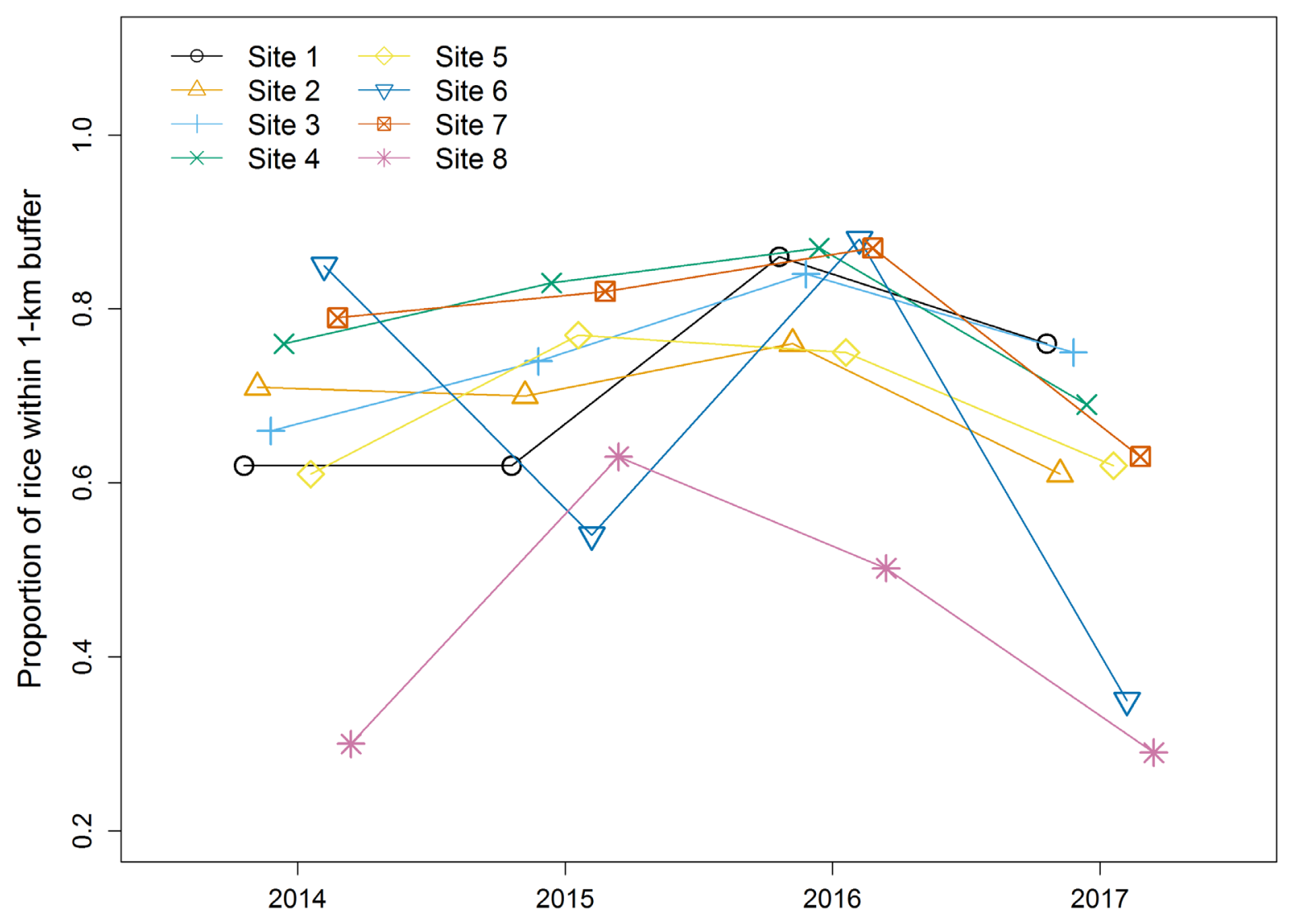

Figure 9. Proportional area of rice growing from 2014-17 at eight sites in the Sacramento Valley, California. Values are based on proportional area where rice was grown within a 1-km buffer of our trap locations in 2017.

\section{Size-Distribution of Captured Snakes}

The size distribution of captured snakes was similar for most sites. The most abundant size class of male snakes was between approximately $550-650 \mathrm{~mm} \mathrm{SVL}$ and $>50 \mathrm{~g}$ mass at most sites. At site 4 , smaller males with an SVL near $400 \mathrm{~mm}$ and a mass of approximately $50 \mathrm{~g}$ were more common, and at site 1 , males near $50 \mathrm{~g}$ mass were the most common (figs. 10 and 11). At sites $1-3,6$, and 8 , there were two peaks in the distribution of SVLs from captured females; one peak near 400-500 mm SVL and another at approximately 700-800 mm SVL (fig. 10). Sites 5 and 7 were notable for the lack of small giant gartersnakes, particularly small females (figs. 10 and 11). At both site 5 and site 7, the most abundant size class of female snakes was $>800 \mathrm{~mm}$ SVL and $>200 \mathrm{~g}$ mass. In contrast, we captured few large female giant gartersnakes at site 4 : the distributions of SVL and mass appear truncated at this site (figs. 10 and 11). Females near 500 $\mathrm{mm}$ SVL and between 50-150 g mass were the most abundant size class at site 4, and we captured few females $>800 \mathrm{~mm} \mathrm{SVL}$ and $>200 \mathrm{~g}$ at this site. Captures of large females were particularly rare at site 4 in 2017. 

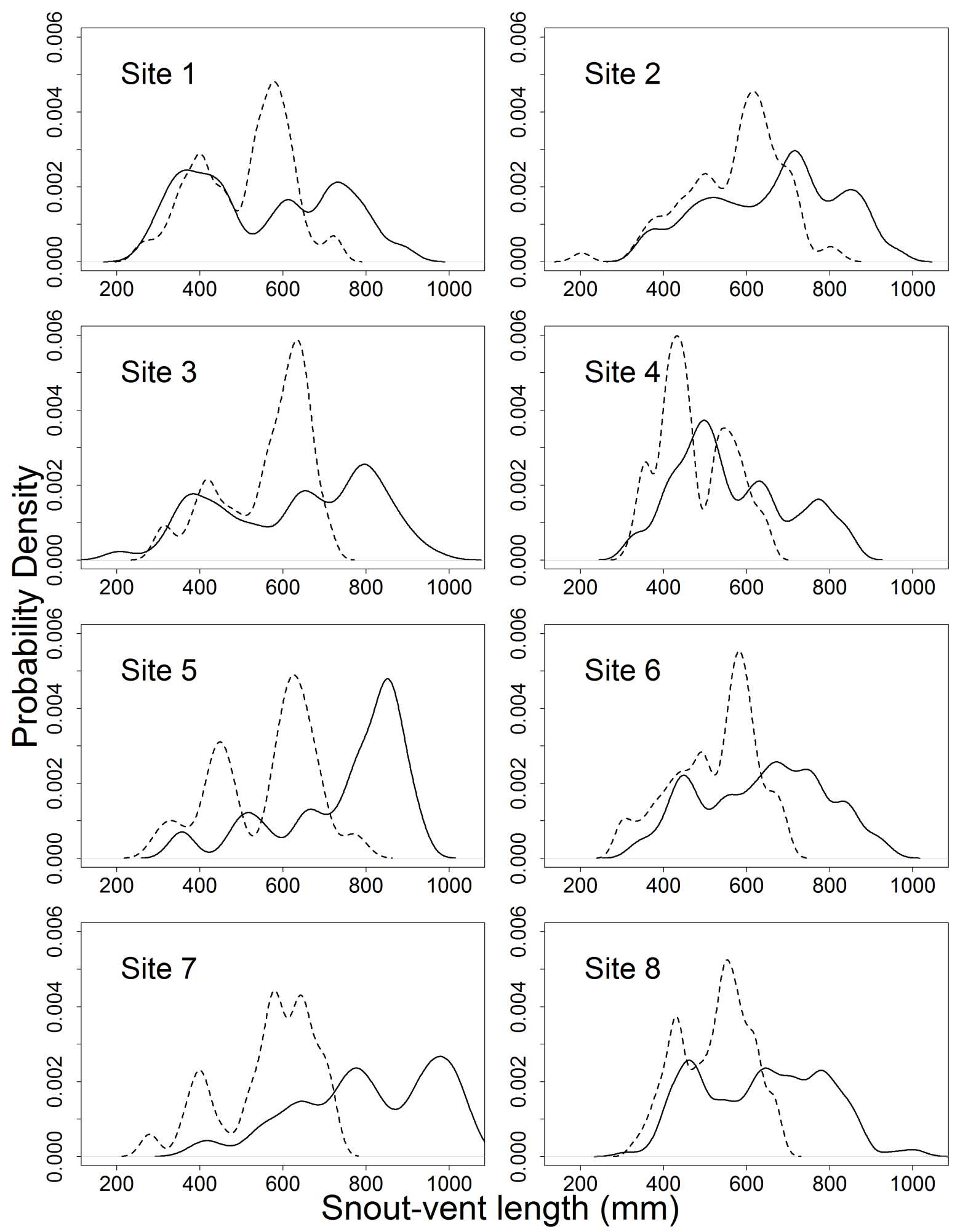

Figure 10. Distribution of snout-vent lengths (SVL) of captured giant gartersnakes by site for eight sites in the Sacramento Valley, California, from 2014-17. Lines are kernel density estimates from observed SVLs. Solid lines represent females, whereas dashed lines represent males. 

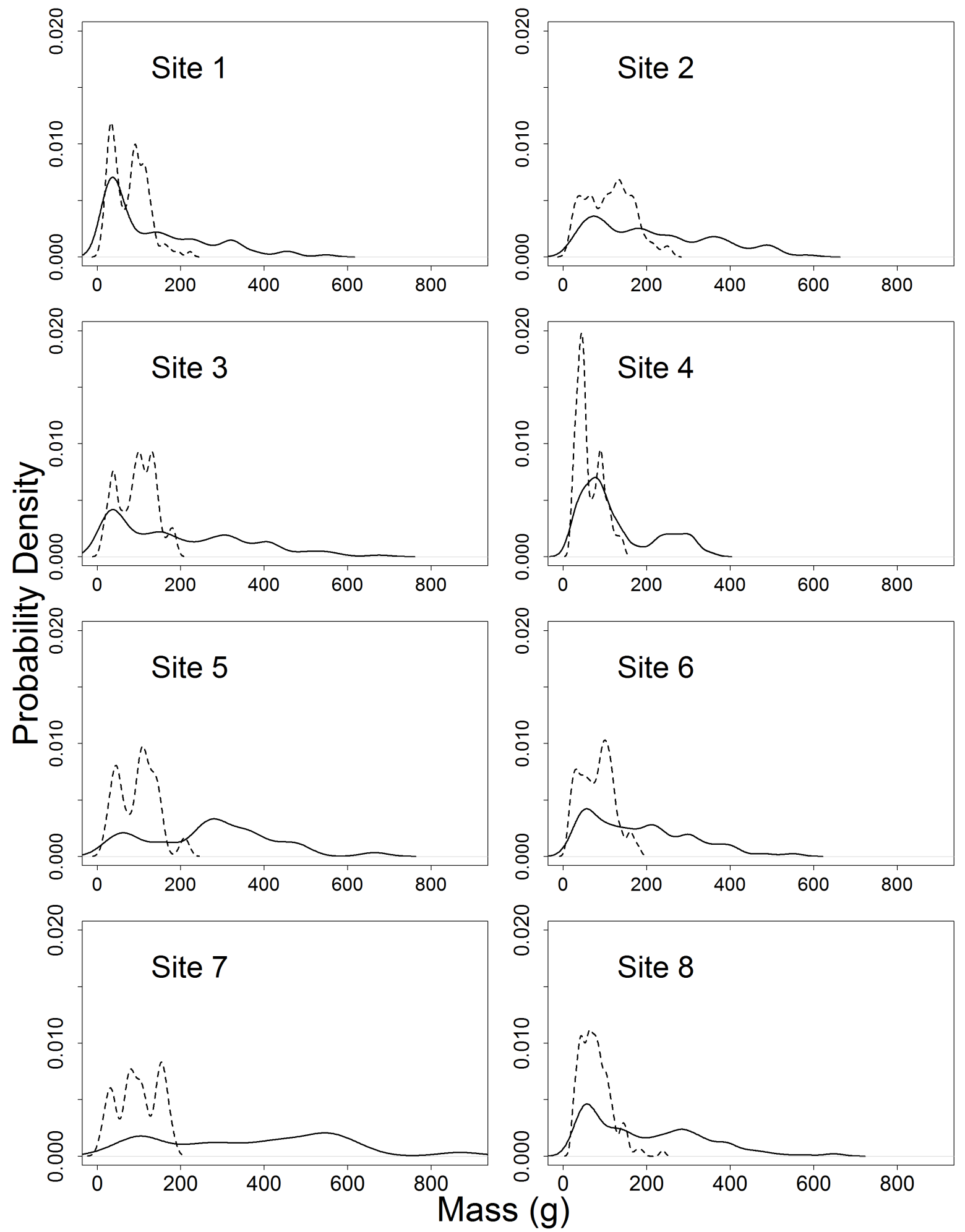

Figure 11. Distribution of the mass $(\mathrm{g})$ of captured giant gartersnakes from eight sites in the Sacramento Valley, California, from 2014-17. Lines are kernel density estimates from observed masses. Solid lines represent females, whereas dashed lines represent males. 


\section{Growth}

We obtained growth data from 192 snakes that were measured in multiple years, including 99 females and 93 males. We measured the SVL of 175 snakes twice, 16 snakes three times, and 1 snake four times, resulting in a total of 210 growth increments. As expected, females reached greater asymptotic SVLs $\left(\mathrm{L}_{\infty}\right)$ than males, but there was only weak evidence that the sexes differ in $k$, which describes the rate at which individuals grow towards their asymptotic SVL (table 9).

Table 9. Parameter estimates from a von Bertalanffy growth model fit to growth data from giant gartersnakes at eight study sites sampled in the Sacramento Valley, 2014-17.

[SD = standard deviation; $2.5=2.5$ th percentile; $97.5=97.5$ th percentile; $\operatorname{Pr}=$ probability $]$

\begin{tabular}{|c|c|c|c|c|c|c|}
\hline \multirow[b]{2}{*}{ Parameter } & \multirow[b]{2}{*}{ Symbol } & \multirow[b]{2}{*}{ Mean } & \multirow[b]{2}{*}{ SD } & \multicolumn{2}{|c|}{ Percentile } & \multirow[b]{2}{*}{$\operatorname{Pr}(m<0)$} \\
\hline & & & & 2.5 & 97.5 & \\
\hline Asymptotic size (female) & $\mathrm{L}_{\infty}$ & 863.5 & 18.4 & 829.6 & 902.0 & -- \\
\hline Growth coefficient & $k$ & 1.8 & 0.4 & 0.9 & 2.7 & -- \\
\hline SD of year on $k$ & $\sigma_{\mathrm{ky}}$ & 0.6 & 0.5 & 0.1 & 1.4 & -- \\
\hline SD of site on $k$ & $\sigma_{\mathrm{ks}}$ & 0.4 & 0.3 & 0.0 & 0.8 & -- \\
\hline $\mathrm{SD}$ of site on $\mathrm{L}_{\infty}$ & $\sigma_{\mathrm{L}}$ & 0.2 & 0.2 & 0.0 & 0.5 & -- \\
\hline $\mathrm{SD}$ (measurement error) & $\sigma_{\mathrm{e}}$ & 33.7 & 2.3 & 29.1 & 38.3 & -- \\
\hline Sex effect on $\mathrm{L}_{\infty}$ (male) & $\mathrm{m}_{\mathrm{L}}$ & -186.5 & 23.6 & -221.6 & -128.5 & 1.0 \\
\hline Sex effect on $k$ & $m_{k}$ & -0.2 & 0.3 & -0.7 & 0.4 & 0.8 \\
\hline
\end{tabular}

We measured 191 annual growth increments from 178 snakes captured and measured in consecutive years, including 93 females and 85 males. Most growth measurements (131/191) came from the 2016-17 interval when trapping effort was highest (table 10). Averaged over a 365 day year, growth rates averaged $0.28 \mathrm{~mm} /$ day (standard deviation $[\mathrm{SD}]=0.22$ ) for females and $0.19 \mathrm{~mm} /$ day $(\mathrm{SD}=0.20)$ for males. Our regression model showed support for a faster growth rate in female snakes than males $\left(\beta_{\text {male }}=-0.27,95\right.$-percent $\mathrm{CRI}=-0.31$ to -0.23$)$ and for a negative relationship between snake SVL and growth rate $\left(\beta_{S V L}=-0.19,-0.21\right.$ to -0.16$)$. There was some support for an interactive effect between sex and SVL on growth rate ( $\beta$ male $\mathrm{SVL}$ $\left.=-0.03,-0.07-0.01 ; \operatorname{Pr}\left[\beta_{\text {male }} \times \mathrm{SVL}<0=0.90\right]\right)$, although the 95-percent credible interval overlapped zero. This interactive effect of sex and SVL on growth rate means that male snakes' growth rate decreased faster with increasing SVL. More of the variation in growth rate was attributable to differences among years $\left(\sigma_{\text {year }}=0.17,0.01-0.55\right)$ than among sites $\left(\sigma_{\text {site }}=0.04\right.$, $7.7 \mathrm{e}-5-0.08)$ or individuals $\left(\sigma_{\text {ind }}=0.02,5.1 \mathrm{e}-6-0.05\right)$. 
Table 10. Growth rates of giant gartersnakes from 2014-17 summarized by site, sex, and year, for eight sites in the Sacramento Valley, California.

\begin{tabular}{lllrcc}
\hline & & & & \multicolumn{2}{c}{ Growth rate (mm/day) } \\
\cline { 5 - 6 } Site & Year & Sex & N & Mean & SD \\
\hline 1 & 2014 & F & 6 & 0.38 & 0.34 \\
1 & 2014 & M & 5 & 0.00 & 0.26 \\
1 & 2015 & F & 2 & 0.20 & 0.04 \\
1 & 2015 & M & 5 & 0.13 & 0.18 \\
1 & 2016 & F & 6 & 0.33 & 0.31 \\
1 & 2016 & M & 14 & 0.20 & 0.12 \\
2 & 2014 & F & 2 & 0.11 & 0.15 \\
2 & 2015 & F & 2 & 0.11 & 0.05 \\
2 & 2015 & M & 1 & -0.06 & -- \\
2 & 2016 & F & 10 & 0.31 & 0.25 \\
2 & 2016 & M & 8 & 0.32 & 0.35 \\
3 & 2014 & F & 2 & 0.07 & 0.08 \\
3 & 2015 & F & 4 & 0.17 & 0.32 \\
3 & 2016 & F & 12 & 0.36 & 0.28 \\
3 & 2016 & M & 7 & 0.09 & 0.06 \\
4 & 2015 & F & 2 & 0.18 & 0.02 \\
4 & 2015 & M & 1 & 0.04 & -- \\
4 & 2016 & F & 2 & 0.32 & 0.04 \\
4 & 2016 & M & 3 & 0.19 & 0.12 \\
5 & 2014 & F & 1 & 0.19 & -- \\
5 & 2015 & F & 2 & 0.29 & 0.15 \\
5 & 2015 & M & 2 & 0.41 & 0.56 \\
5 & 2016 & F & 5 & 0.13 & 0.16 \\
5 & 2016 & M & 2 & 0.11 & 0.03 \\
6 & 2015 & F & 9 & 0.33 & 0.19 \\
6 & 2015 & M & 8 & 0.06 & 0.21 \\
6 & 2016 & F & 11 & 0.35 & 0.18 \\
6 & 2016 & M & 10 & 0.25 & 0.19 \\
7 & 2015 & F & 2 & 0.01 & 0.05 \\
7 & 2015 & M & 2 & 0.24 & 0.22 \\
7 & 2016 & F & 2 & 0.19 & 0.01 \\
\hline & 2016 & M & 3 & 0.05 & 0.06 \\
8 & 2015 & F & 2 & 0.15 & 0.07 \\
\hline & 2016 & F & 18 & 0.31 & 0.19 \\
N & & 18 & 0.26 & 0.13 \\
\hline & & & &
\end{tabular}




\section{Health Assessments}

Health assessments were conducted for 22 snakes in 2014, 37 snakes in 2015, 20 snakes in 2016, and 80 snakes in 2017. Health assessments in 2017 were only conducted on female snakes that were large enough that they could be reproductively mature (greater than $500 \mathrm{~mm}$ SVL except one snake with an SVL of $435 \mathrm{~mm}$ ), whereas health assessments from 2014-16 were primarily conducted on snakes that were part of a radio-telemetry study (Reyes and others, 2017), and thus snakes had to have a mass greater than or equal to $200 \mathrm{~g}$ to be examined. Means and standard deviations for health parameters by year at each site are presented in tables 11 and 12. The data did not show any clear signs of poor health in our sample of giant gartersnakes. The values for the blood and plasma biochemistry parameters measured in this study are comparable to the baseline values from "apparently healthy" giant gartersnakes reported by Wack and others (2012). We did not evaluate trends in health parameters because of the small sample sizes from 2014-16. Continued examination of larger numbers of snakes would allow us to evaluate if the health of snakes varies among sites and over time and if snake health is related to landscape level variables such as the amount of active rice growing. 
Table 11. Summary of blood cell counts, hemoglobin, and hematocrit of female giant gartersnakes of reproductive size by site and year for eight sites in the Sacramento Valley, California, 2014-17.

[For blood parameters, values are means followed by standard deviations in parentheses. WBC, white blood cells $\left(\times 10^{3} / \mu \mathrm{L}\right)$; $\mathrm{RBC}$, red blood cells $\left(\times 10^{6} / \mu \mathrm{L}\right)$; $\mathrm{HgB}$, hemoglobin (g/dL); PCV, packed cell volume (\%). White blood cell types (for example, heterophils - azurophils) are \% of counted cells]

\begin{tabular}{|c|c|c|c|c|c|c|c|c|c|c|c|c|}
\hline Site & Year & $\mathbf{N}$ & WBC & RBC & $\mathrm{HgB}$ & PCV & Heterophils & Lymphocytes & Monocytes & Eosinophils & Basophils & Azurophils \\
\hline 1 & 2014 & 2 & $6.8(6.8)$ & $0.8(0)$ & $8.4(0.6)$ & $25.5(2.1)$ & $8.5(4.9)$ & $36.9(51.1)$ & $0(0)$ & $0(0)$ & $3.5(0.7)$ & $13.5(2.1)$ \\
\hline 1 & 2015 & 6 & $4.5(3.3)$ & $0.9(0.3)$ & $8.7(2.3)$ & $26.7(6.7)$ & $11.8(5)$ & $77.7(9.8)$ & $1.5(1.6)$ & $0(0)$ & $2.2(2.4)$ & $6.8(8.4)$ \\
\hline 1 & 2016 & 4 & $11.5(6.5)$ & $0.6(0.2)$ & $9.1(0.1)$ & $27.3(1)$ & $8.8(8.5)$ & $71(10.9)$ & $1.3(1.5)$ & $0(0)$ & $2(0.8)$ & $17(6.3)$ \\
\hline 1 & 2017 & 7 & $5.9(2)$ & $0.6(0.2)$ & $8(2.3)$ & $25.9(7.6)$ & $12.7(8.3)$ & $64.7(11.1)$ & $0.7(1)$ & $0.9(1.2)$ & $5.3(3.7)$ & $15.7(10.5)$ \\
\hline 2 & 2014 & 4 & $8.3(3.7)$ & $0.9(0.1)$ & $10(0.5)$ & $30(3.2)$ & $5.3(3.4)$ & $74.3(11.1)$ & $1.3(1.3)$ & $0(0)$ & $5.8(2.1)$ & $13.5(7.9)$ \\
\hline 2 & 2015 & 4 & $4.9(4.2)$ & $0.9(0.2)$ & $10.5(2.4)$ & $31(8.2)$ & $15.3(7.3)$ & $64.3(19.6)$ & $2.5(2.4)$ & $0(0)$ & $1.3(1.5)$ & $4.5(7)$ \\
\hline 2 & 2016 & 2 & $2.9(1.2)$ & $0.6(0.2)$ & $9.8(2.2)$ & $29(5.7)$ & $30.5(16.3)$ & $69.5(16.3)$ & $0(0)$ & $0(0)$ & $0(0)$ & $0(0)$ \\
\hline 2 & 2017 & 12 & $5.9(4.7)$ & $0.7(0.2)$ & $8.1(1.5)$ & $24.3(3.4)$ & $8.7(4)$ & $71.8(8.2)$ & $0.2(0.6)$ & $1.7(5.8)$ & $3.3(4)$ & $16.9(8.7)$ \\
\hline 3 & 2014 & 6 & $4.5(1.7)$ & $1.2(1.5)$ & $8.4(0.9)$ & $24.8(1.6)$ & $15.3(12.2)$ & $74.7(8.7)$ & $0.5(0.5)$ & $0.2(0.4)$ & $3(3.8)$ & $6.3(6.9)$ \\
\hline 3 & 2015 & 4 & $7.9(5.4)$ & $0.8(0.2)$ & $7.6(1.9)$ & $23.8(5.6)$ & $15.5(6.2)$ & $73.5(5.7)$ & $3.3(4.5)$ & $0(0)$ & $3.5(2.5)$ & $4.3(5.1)$ \\
\hline 3 & 2016 & 1 & 12.9 (NA) & 1 (NA) & 10.1 (NA) & 34 (NA) & 18 (NA) & 82 (NA) & 0 (NA) & 0 (NA) & 0 (NA) & 0 (NA) \\
\hline 3 & 2017 & 14 & $6.7(4.7)$ & $0.6(0.2)$ & $7.8(2.5)$ & $23.6(7.7)$ & $9.9(9.4)$ & $67.6(11.5)$ & $0.8(2.9)$ & $0(0)$ & $1(1.4)$ & $20.7(11.5)$ \\
\hline 4 & 2015 & 5 & $4.3(1.9)$ & $0.8(0.2)$ & $9.5(1.4)$ & $29.6(5.1)$ & $14(8.7)$ & $77.8(5.5)$ & $0(0)$ & $0(0)$ & $5.4(5.5)$ & $2.4(2.9)$ \\
\hline 4 & 2016 & 2 & $1.3(1.6)$ & $0.6(0.1)$ & $7.6(0.1)$ & $22.5(0.7)$ & $9.5(0.7)$ & $73.5(2.1)$ & $0.5(0.7)$ & $0(0)$ & $6.5(2.1)$ & $10(0)$ \\
\hline 4 & 2017 & 11 & $3.8(2.2)$ & $0.8(0.2)$ & $8.2(1.1)$ & $25.5(4.1)$ & $6.5(3.8)$ & $77.2(6.6)$ & $0(0)$ & $0(0)$ & $2.5(3.4)$ & $13.8(7.6)$ \\
\hline 5 & 2014 & 5 & $6.7(5.3)$ & $0.7(0.1)$ & $9.1(2.5)$ & $27.4(7.5)$ & $8.8(4.1)$ & $70.6(10.4)$ & $1.4(0.9)$ & $0.2(0.4)$ & $4(2.4)$ & $15.8(11)$ \\
\hline 5 & 2015 & 5 & $7.3(7.8)$ & $0.7(0.2)$ & $7.4(2.9)$ & $22.8(7.9)$ & $15(4.7)$ & $75.6(11.1)$ & $0.4(0.9)$ & $0(0)$ & $1.8(2)$ & $7.2(10.5)$ \\
\hline
\end{tabular}




\begin{tabular}{|c|c|c|c|c|c|c|c|c|c|c|c|c|}
\hline Site & Year & $\mathbf{N}$ & WBC & RBC & $\mathrm{HgB}$ & PCV & Heterophils & Lymphocytes & Monocytes & Eosinophils & Basophils & Azurophils \\
\hline 5 & 2016 & 4 & $12(5.7)$ & $0.7(0.3)$ & $8.2(2.9)$ & $25.5(9.1)$ & $9.5(10.6)$ & $70.3(16.3)$ & $0.5(1)$ & $0(0)$ & $2(0.8)$ & $17.8(5.7)$ \\
\hline 5 & 2017 & 8 & $5(3.4)$ & $1.4(2.3)$ & $7.6(2.2)$ & $22.1(6.7)$ & $3.8(2.7)$ & $70(14.6)$ & $1(2.4)$ & $0.8(2.1)$ & $2.8(3.5)$ & $21.8(14.9)$ \\
\hline 6 & 2015 & 4 & $4.6(2.1)$ & $0.8(0.4)$ & $7.8(3)$ & $24(9.7)$ & $21.8(15.1)$ & $77.3(13.6)$ & $0(0)$ & $0(0)$ & $0.8(1.5)$ & $0.3(0.5)$ \\
\hline 6 & 2016 & 1 & 3.4 (NA) & 0.7 (NA) & 10.4 (NA) & 32 (NA) & 12 (NA) & 87 (NA) & 0 (NA) & 0 (NA) & 0 (NA) & 1 (NA) \\
\hline 6 & 2017 & 10 & $4.7(3.7)$ & $0.8(0.2)$ & $9.1(2.2)$ & $27.6(6.1)$ & $12.2(5.8)$ & $59(16)$ & $0(0)$ & $0(0)$ & $0.6(0.8)$ & $28.1(15.8)$ \\
\hline 7 & 2015 & 4 & $9.6(5.1)$ & $0.8(0.3)$ & $8.7(0.7)$ & $27.5(1.7)$ & $16(12.1)$ & $72.3(6.7)$ & $1.3(1.9)$ & $0(0)$ & $1.8(2.1)$ & $8.8(10.3)$ \\
\hline 7 & 2016 & 2 & $6.3(3)$ & $0.9(0.2)$ & $8.9(1.8)$ & $30(8.5)$ & $8(2.8)$ & $85.5(0.7)$ & $0.5(0.7)$ & $0(0)$ & $1.5(2.1)$ & $4.5(0.7)$ \\
\hline 7 & 2017 & 4 & $5.4(5.8)$ & $0.6(0.2)$ & $7.8(2.3)$ & $23(6.7)$ & $5(2.9)$ & $77.5(3.1)$ & $0(0)$ & $0.3(0.5)$ & $0.5(0.6)$ & $16(4.2)$ \\
\hline 8 & 2014 & 5 & $8.6(4.3)$ & $0.9(0.5)$ & $9.1(2)$ & $27.8(5.4)$ & 13 (11.6) & $76.8(10.5)$ & $0.4(0.5)$ & $0.2(0.4)$ & $3(3.6)$ & $7.2(7.9)$ \\
\hline 8 & 2015 & 5 & $13.1(4.9)$ & $0.9(0.2)$ & $10(2.3)$ & $\begin{array}{c}32.2(6.5) \\
26.5\end{array}$ & $10.2(6.4)$ & $75.6(6.7)$ & $2.6(0.9)$ & $0(0)$ & $0.2(0.4)$ & $8.4(9.6)$ \\
\hline 8 & 2016 & 4 & $6.5(4.8)$ & $0.5(0.3)$ & $9(4.5)$ & $(12.5)$ & $6.8(3)$ & $84.8(11.9)$ & $0(0)$ & $0(0)$ & $2.3(3.3)$ & $6.3(9.5)$ \\
\hline 8 & 2017 & 14 & $5.1(3.2)$ & $0.7(0.2)$ & $8(1.7)$ & $23.6(6.6)$ & $7(4.2)$ & $69.2(12.2)$ & $0.3(0.8)$ & $0(0)$ & $2.2(2)$ & $21.1(11.8)$ \\
\hline
\end{tabular}


Table 12. Summary of blood solutes of female giant gartersnakes of reproductive size by site and year for eight sites in the Sacramento Valley, California, 2014-17.

[For blood parameters, values are means followed by standard deviations in parentheses. P. protein, plasma protein (g/dL); AST, aspartate aminotransferase (IU/L); CK, creatinine kinase (IU/L); UA, uric acid (mg/dL); glucose (mg/dL); Ca, calcium (mg/dL); P, phosphorus (mg/dL); T. protein, total protein (g/dL); albumin $(\mathrm{g} / \mathrm{dL})$; globulin $(\mathrm{g} / \mathrm{dL}), \mathrm{K}$, potassium $(\mathrm{mmol} / \mathrm{dL}) ; \mathrm{Na}$, sodium $(\mathrm{mmol} / \mathrm{dL})]$

\begin{tabular}{|c|c|c|c|c|c|c|c|c|c|c|c|c|c|}
\hline Site & Year & P. protein & AST & CK & UA & Glucose & $\mathrm{Ca}$ & $\mathbf{P}$ & T. protein & Albumin & Globulin & $\mathrm{K}$ & $\mathrm{Na}$ \\
\hline 1 & 2014 & $8.1(2.1)$ & $15.5(0.7)$ & $449.5(289.2)$ & $6.1(0.3)$ & $85.5(20.5)$ & $16.1(0.1)$ & $8.1(3.3)$ & $6.1(0.5)$ & $1.6(0.3)$ & $4.4(0.3)$ & $3.6(0.4)$ & $154.5(0.7)$ \\
\hline 1 & 2015 & $3.7(1.2)$ & $19(13.2)$ & $311(215.1)$ & $6.1(2)$ & $76(10.7)$ & $13.4(2.2)$ & $4.1(1.1)$ & $3.6(0.9)$ & $1.1(0.2)$ & $1.4(1.5)$ & $4.8(1)$ & $155(2.8)$ \\
\hline 1 & 2016 & $5.7(0.6)$ & $18.5(1.7)$ & $309.5(213.9)$ & $11.4(6.8)$ & $97.3(7.9)$ & $14.4(1.2)$ & $5(1.3)$ & $4.9(0.6)$ & $1.2(0.1)$ & $3.6(0.6)$ & $5.9(1.3)$ & $154.8(6.1)$ \\
\hline 1 & 2017 & $4.9(1.1)$ & $11.3(0.8)$ & $429.5(255.5)$ & $7.2(3.3)$ & $95.3(61.1)$ & $13.2(0.3)$ & $4.9(1.6)$ & $4.1(0.3)$ & $1.2(0.2)$ & $2.9(0.3)$ & $5(0.5)$ & $152.8(5.9)$ \\
\hline 2 & 2014 & $5.6(0.4)$ & 20.5 (7.9) & $467.8(72.9)$ & $3.5(1.6)$ & $111(31.4)$ & $14.9(0.9)$ & $5(0.7)$ & $5(0.3)$ & $1.4(0.2)$ & $3.5(0.2)$ & $5.7(0.9)$ & $159.5(4)$ \\
\hline 2 & 2015 & $5.9(0.6)$ & $13.3(4.8)$ & $239.8(105.8)$ & $5.7(4.9)$ & $59.3(18.6)$ & $15.3(0.6)$ & $5(1.8)$ & $5.1(0.4)$ & $1.4(0.1)$ & $3.7(0.5)$ & $5(1.1)$ & $162.5(4.5)$ \\
\hline 2 & 2016 & $6.7(2.4)$ & $13.5(2.1)$ & $422(314)$ & $14.1(3)$ & $79.5(9.2)$ & $6.3(8.9)$ & $7(4.2)$ & $5.1(0.9)$ & $1.6(0.6)$ & $3.5(0.4)$ & $5.3(0.1)$ & $161.5(2.1)$ \\
\hline 2 & 2017 & $5(0.9)$ & $12.7(2.6)$ & $468(321.5)$ & $6.5(7.5)$ & $82.5(37.1)$ & $13.9(1.2)$ & $5.5(1.1)$ & $4.3(0.4)$ & $1.2(0.1)$ & $2.8(1.3)$ & $5.3(0.3)$ & $154.3(3.3)$ \\
\hline 3 & 2014 & $4.9(0.4) 2$ & 27.5 (17.9) & $1043.2(1021)$ & $3.8(1.1)$ & 77.7 (12.8) & $14.6(1.2)$ & $5.7(1.3)$ & $4.6(0.4)$ & $1.2(0.1)$ & $3.4(0.4)$ & $4.4(0.5)$ & $158.7(7.1)$ \\
\hline 3 & 2015 & $4.7(1)$ & $14(7.3)$ & $432.8(230.8)$ & $4(2.1)$ & $63.3(17.8)$ & $15.8(0.6)$ & $6.8(1.6)$ & $4.2(0.8)$ & $1.1(0.3)$ & $1.8(2.1)$ & $5.7(1.6)$ & $157.8(0.5)$ \\
\hline 3 & 2016 & 5.6 (NA) & 14 (NA) & 117 (NA) & 8.2 (NA) & 119 (NA) & 13.8 (NA) & 2.8 (NA) & 5.2 (NA) & 1.1 (NA) & 4.1 (NA) & 4.7 (NA) & 160 (NA) \\
\hline 3 & 2017 & $4.9(1.6)$ & $13.7(4.3)$ & 850.7 (1047.5) & $5(3)$ & $85.9(29.4)$ & $13.1(1)$ & $5.5(1.8)$ & $4.3(0.7)$ & $1(0.3)$ & $1.5(1.8)$ & $5.5(1)$ & $155.9(3.7)$ \\
\hline 4 & 2015 & $6.2(1)$ & $16.6(5.4)$ & $571.6(485.7)$ & $4.1(2.1)$ & $64.6(23.8)$ & $15.8(0.6)$ & $6.4(1)$ & $4.7(0.6)$ & $1.3(0.1)$ & $3.4(0.5)$ & $5.3(0.7)$ & $160(3.7)$ \\
\hline 4 & 2016 & $6.5(0.1)$ & $18.5(3.5)$ & 1587 (1548.6) & $8.8(3.5)$ & $62.5(17.7)$ & $15.7(0.6)$ & $9.2(5.8)$ & $5.1(0.3)$ & $1.8(0.4)$ & $3.4(0.6)$ & $6.8(0.3)$ & $160.5(0.7)$ \\
\hline 4 & 2017 & $5.3(1.1)$ & $16.7(13)$ & $457.9(250.1)$ & $3.9(1.5)$ & $79.6(22.7)$ & $13.8(0.7)$ & $4.6(1)$ & $4.7(0.4)$ & $1.3(0.1)$ & $3.4(0.3)$ & $5.2(0.5)$ & $155.4(5.1)$ \\
\hline 5 & 2014 & $5.8(0.7) 2$ & $24.4(25.8)$ & $940(1073.1)$ & $4(2.5)$ & $77(18.5)$ & $15(1.1)$ & $5.2(0.8)$ & $4.9(1.2)$ & $1.2(0.2)$ & $3.3(1.9)$ & $4.5(0.8)$ & $159.6(7.5)$ \\
\hline 5 & 2015 & $5.4(0.8)$ & $15.8(3.6)$ & 470 (357) & $3.7(2)$ & $72.8(13.1)$ & $14.4(1.2)$ & $5.6(3.1)$ & $4.6(0.4)$ & $1.3(0.1)$ & $3.3(0.4)$ & $5.2(0.6)$ & $162.4(3.8)$ \\
\hline 5 & 2016 & $4.9(1.3)$ & $21.5(5.8)$ & $698.5(930.8)$ & $7.9(7)$ & $80.3(7.1)$ & $13.9(1.2)$ & $5.5(0.8)$ & $4.7(0.8)$ & $1.2(0.1)$ & $2.9(1.9)$ & $5.2(0.8)$ & $158(2.9)$ \\
\hline 5 & 2017 & $5.2(1.3)$ & $12.6(5.1)$ & $269.3(207.8)$ & $8.3(8.2)$ & $73.9(22.3)$ & $13.8(1.3)$ & $4.8(0.7)$ & $4.8(1.2)$ & $1.1(0.3)$ & $2.5(2.2)$ & $5.3(0.6)$ & $155.4(4.1)$ \\
\hline
\end{tabular}




\begin{tabular}{|c|c|c|c|c|c|c|c|c|c|c|c|c|c|}
\hline Site & Year & P. protein & AST & $\mathrm{CK}$ & UA & Glucose & $\mathrm{Ca}$ & $\mathbf{P}$ & T. protein & Albumin & Globulin & $\mathrm{K}$ & $\mathrm{Na}$ \\
\hline 6 & 2015 & $6.1(1.7)$ & $17.8(6.7)$ & $1452.8(975.8)$ & $6.4(3)$ & $49.8(21.7)$ & $15.4(1.5)$ & $6.2(2.4)$ & $4.7(1.2)$ & $1.4(0.3)$ & $3.3(0.9)$ & $4.6(0.1)$ & $156(2.9)$ \\
\hline 6 & 2016 & 4.6 (NA) & 22 (NA) & 415 (NA) & 10.2 (NA) & 75 (NA) & 13.6 (NA) & 5.4 (NA) & 5.1 (NA) & 1.4 (NA) & 3.7 (NA) & $5(\mathrm{NA})$ & 167 (NA) \\
\hline 6 & 2017 & $4.5(1.1)$ & $12.6(6.2)$ & $464.5(564.9)$ & $5.1(2.6)$ & $80.8(37.8)$ & $13(1)$ & $4(0.5)$ & $4.1(0.9)$ & $1.2(0.3)$ & $1.7(1.8)$ & $4.7(0.4)$ & $152.3(2.5)$ \\
\hline 7 & 2015 & $5.4(0.5)$ & $12.8(3.4)$ & $440.5(359.2)$ & $5.6(2.3)$ & $83.3(14.6)$ & $14.9(0.8)$ & $4.6(0.8)$ & $5(0.4)$ & $1.3(0.1)$ & $3.7(0.3)$ & $4.7(0.7)$ & $156.5(6)$ \\
\hline 7 & 2016 & $6.7(1.8)$ & $35.5(19.1)$ & $651(343.7)$ & $9(3.4)$ & $80.5(14.8)$ & $15.4(1.1)$ & $7.3(3.5)$ & $4.6(0.3)$ & $1.4(0.2)$ & $3.3(0)$ & $4.2(0.6)$ & $162(2.8)$ \\
\hline 7 & 2017 & $4.2(0.3)$ & $11.3(1.5)$ & $388(266)$ & $11.2(8.6)$ & $106.8(59)$ & $13.7(0.7)$ & $5.4(0.8)$ & $3.9(0.4)$ & $1.2(0.2)$ & $2.1(1.4)$ & $4.6(0.2)$ & $153.8(8.1)$ \\
\hline 8 & 2014 & $5.1(0.8)$ & $35.8(25.9)$ & $581.6(442.4)$ & $3.1(0.8)$ & $96.6(17.8)$ & $16(0.2)$ & $6.1(1)$ & $4.8(0.7)$ & $1.2(0.2)$ & $3.8(0.5)$ & $3.8(0.5)$ & $159.8(4.6)$ \\
\hline 8 & 2015 & $5.5(0.9)$ & $25(29.7)$ & $928.4(1124)$ & $6(1.8)$ & $74.8(21.3)$ & $16(0.3)$ & $5.8(1.6)$ & $4.9(0.9)$ & $1.3(0.2)$ & $3.7(0.7)$ & $4.2(0.5)$ & $157.2(4.8)$ \\
\hline 8 & 2016 & $6.6(1.6)$ & $17.8(3.8)$ & $415.8(197.3)$ & $6.9(2)$ & $86.3(19.7)$ & $15.6(1.1)$ & $7.7(2.4)$ & $4.7(0.8)$ & $1.4(0.2)$ & $3.4(0.6)$ & $5(0.4)$ & $158.3(3.3)$ \\
\hline$\underline{8}$ & 2017 & $5(1)$ & $15.2(6.4)$ & $729.5(938.6)$ & $8.7(6.3)$ & $74.1(25.9)$ & $15.7(3.8)$ & $6(2)$ & $4.3(0.5)$ & $1.2(0.3)$ & $2.3(1.5)$ & $5.4(0.9)$ & $152.9(4.5)$ \\
\hline
\end{tabular}




\section{Discussion}

The results from 4 years of sampling at these eight sites ( 2 years of preliminary sampling, 2 years of full demographic sampling) demonstrate that we can obtain a wealth of data on the reproduction, survival, somatic growth, and abundance of giant gartersnakes inhabiting canals adjacent to rice agriculture by intensively trapping the same areas in consecutive years. It is especially promising that the increased sampling effort in 2016 and 2017 (the first 2 years of sampling designed explicitly for this study) led to more captures and recaptures of giant gartersnakes and more precise estimates of abundance, population growth, and survival. Although some sites inhabited by giant gartersnakes have been the subject of long-term studies (for example, Hansen and others, 2015; Rose and others, 2018a,b), this study represented, to the best of our knowledge, the first project to collect simultaneous, long-term data on all aspects of giant gartersnake demography from multiple populations in privately owned, rice-growing areas. The opportunity to collect more data on variation in rice growing over time and among sites would allow us to evaluate whether and how the amount of rice growing at a site relates to snake health and demographic rates of interest, such as somatic growth, survival, fecundity, the proportion of gravid females, and population growth. Because the study has been discontinued, we are unable to further investigate these questions at this time.

We observed changes in the amount of rice grown at a site over time. Although most sites exhibited a peak in rice production in 2016, the fluctuations in rice growing were not synchronous among sites. Some sites exhibited small changes between years, whereas sites 6 and 8 had more dramatic shifts in the amount of rice grown. The observed range of the area under active rice cultivation was 30-90 percent of the area within a 1-km buffer of our traplines. We believe this range is likely biologically relevant to giant gartersnakes for two reasons. First, given the relationship between giant gartersnake presence and rice growing, it is unlikely that large populations of giant gartersnakes (that is, those amenable to demographic sampling) would persist in agricultural areas with no or very little rice (Halstead and others, 2010; Hansen and others, 2017). Second, previous research with radio-tracked adult (mostly female) giant gartersnakes at these sites has linked the area of rice within an individual's home range to its survival rate (Reyes and others, 2017). Therefore, because the variation in the proportion of rice cultivation observed at these sites in the recent past appeared to influence individual survival, we expect the variation in rice cultivation observed in this study to potentially affect population level parameters such as mean survival, abundance, and population growth. Because of their proximity, the 1-km buffers overlap for sites 3 and 5 and for sites 7 and 8 . Future analyses should account for the non-independence of the rice-growing conditions at these sites and for the dispersal of individuals between sites (see discussion of size distributions, below). 
One of the most encouraging signs from this study was the rate of annual recaptures. Even though full demographic sampling only began in 2016, on average, 19 percent of snakes were captured in 2 or more years. Given that we estimate the annual apparent survival probability to be between 0.2 and 0.6 depending on the site, this means we are recapturing a large proportion of the marked snakes that survive (and remain on site) each year. Also, we emphasize that the majority of snakes ( 75.9 percent) were captured for the first time in 2016 (47.5 percent) or 2017 (28.4 percent) —when sampling effort increased at the beginning of this study - and therefore could only have been recaptured once (if first caught in 2016) or zero times (if first caught in 2017). It is also notable that the rate of immediate annual recaptures (that is, a snake is recaptured the next year after its initial capture) was higher in the 2 most recent years, when sampling effort was greater than the first 2 years. Based on these trends, it is likely that a large proportion of the marked snakes that are available for capture would be recaptured in future years, as they are exposed to additional years of sampling. For example, of the 120 snakes first captured in 2015, 43 (35.8 percent) were recaptured in one (34 snakes) or both ( 9 snakes) of the subsequent years. The precision of annual survival estimates improves with the number of marked individuals, the proportion of marked individuals that are subsequently recaptured (recapture probability), and, for longer-lived organisms with low recapture probabilities, the duration of the study (Halstead and others, 2015a). Thus, had the study continued, the large number of marked individuals combined with the high recapture rates would have allowed for more precise estimates of survival, which is a key vital rate for assessing population viability. Also, by estimating how survival varies among sites and years, we would have been able to test for a relationship between the amount of aquatic habitat available (that is, the area under active rice growing) and annual survival. Estimating site by year differences in survival will be important for assessing the importance of aquatic habitat availability for giant gartersnake survival. Comparing how survival varies in relation to changes in the amount of rice grown at a site would allow us to assess if increasing or decreasing the area under rice cultivation has an effect on the survival of giant gartersnakes inhabiting canals near agricultural fields.

Given the change in sampling intensity from 2014-15 (before the current study began) to 2016-17, we caution against interpreting population trends from these data. For example, although the mean estimated abundance of giant gartersnakes at site 1 appeared to increase from 55 snakes in 2015 to 101 snakes in 2016, we more than doubled our sampling effort in 2016 compared to 2015, and our traps covered a larger area in 2016 as well. Given this change in the sampling effort, only the estimates of population growth for 2014-15 and 2016-17 should be interpreted, and we cannot draw conclusions about population growth from 2015-16. Our estimates of population growth were more precise for 2016-17 than 2014-15, owing to the greater sampling effort in the later years, which improved estimates of abundance. Our results suggest future studies sample the same area at each site with similar trapping effort (as was done in 2016-17). This would allow robust estimates of a time series of giant gartersnake abundance, survival, somatic growth rate, and population growth at each site. These population level parameters could then be compared to the rice growing conditions at each site to see whether and how they are influenced by the availability of aquatic habitat. 
Based on size distributions of captured snakes, five of the sites appeared to have demographically similar populations (sites 1-3,6, and 8). The size distribution was truncated at site 4, which had few large snakes, especially in 2017. The truncated size distribution at site 4 in 2017 could indicate a lack of older adult snakes ( $>3$ years old). The reduced number of large female snakes at site 4 in 2017 could have been caused by delayed sampling. Due to low water levels in site 4's canals in spring 2017, we did not begin sampling until June. Large gravid females might have lower capture probability at this time if they reduce foraging activity prior to parturition. Alternatively, it is possible that growth is much slower at site 4, and the small size of snakes is not indicative of a population dominated by young snakes. We do not think this is likely because variation in growth rate among sites was low, and site 4 had similar growth rates to other sites. It would be interesting to track the size distribution at site 4 in the coming years to see if snakes present in 2016 and 2017 survive and grow to become large adults or if the size distribution remains truncated and dominated by small individuals. In contrast, sites 5 and 7 had few small snakes, and large females were proportionally more common. The lack of small snakes raises concerns that recruitment is low at these sites, and the population is sustained by the continued survival of large, old adult snakes. The lack of small snakes at sites 5 and 7 is especially interesting considering the proximity of these sites to sites 3 and 8 , respectively, which exhibit a more even size distribution with both small and large females. Indeed, sites 3 and 5 are close enough that two snakes moved from site 3 to site 5, and yet the two sites differ dramatically in the size distribution of snakes living there. Given the relatively low survival rate of giant gartersnakes (Rose and others, 2018a), low recruitment, even for a short period, would be expected to lead to a population-level decline in the near term. If recruitment does not increase at sites 5 and 7 in the next few years, and small giant gartersnakes remain scarce, it could signify that the populations of giant gartersnakes at these sites are declining and are at risk of extirpation. 
Although we cannot capture neonate snakes, sampling from mid-April to mid-July is advantageous for studying giant gartersnake demography for a few reasons. Sampling during the "post-reproductive" period after females have begun giving birth is unlikely to result in substantial data on neonate survival because neonate captures and re-captures of snakes marked as neonates are rare (Rose and others, 2018a). The low recapture rate of neonate snakes has been documented for other snake species as well (Pike and others, 2008; Bauwens and Claus, 2018). Additionally, while captures of neonates are rare, sampling intensively while neonates are present exposes neonates to trap-related mortality. These small snakes can become stuck in the mesh of funnel traps while trying to escape, and it is imperative to minimize the risk that our sampling negatively affects populations of this threatened species. Also, by sampling only before parturition takes place, rather than both before and after parturition, we avoid one potential violation of the population closure assumption necessary to fit CMR models. If we sampled both before and after females give birth, we would potentially capture neonate snakes later in the summer that were not available for capture earlier in the sampling period. This influx of neonates could distort our estimates of abundance. By sampling during the same, pre-reproductive period each year, we remove this potentially confounding factor and obtain more consistent estimates of abundance, survival, and population growth each year. Finally, sampling during the "prereproductive" period, before parturition (birth), allows us to examine females and record their reproductive status and, if gravid, litter size. The proportion of females that are gravid and the litter size of gravid females are important demographic variables, and with additional data could allow us to evaluate whether and how variation in these reproductive parameters is related to the amount of rice grown at a site. Although we cannot directly estimate the pulse of neonates that enter the population in the late summer, we can measure the cohort of yearling snakes that appear the following spring. We also can calculate the expected reproductive output of the females in a population, based on observed relationships between female size and fecundity, and use the expected reproductive output in one year, combined with the abundance of yearling snakes the following year, to estimate neonate survival (Rose and others, 2018a).

The probability a female was gravid was not related to the date of examination in 2017 . This indicates that mid-May to mid-July is a good time period during which to examine females' reproductive status. It is late enough that ovulated eggs or developing embryos are visible on Xrays, but early enough that females have not begun to give birth. The first full year in which females were explicitly sampled to examine their reproductive status was 2017. Future examination of a large sample of potentially reproductive females each year would allow us to estimate annual variation in the proportion of females that are gravid and in the fecundity (litter size) of those females. We could also examine if spatial and temporal variation in these reproductive variables is related to environmental conditions such as the amount of rice grown at a site each year.

The timing of reproduction can vary among populations in natricine snakes (Seigel and Ford, 2001). An interesting comparison for giant gartersnakes is the viperine snake (Natrix maura), a semi-aquatic species in the Natricinae sub-family native to southwestern Europe and northwestern Africa that inhabits rice growing regions in Spain. Reproduction is delayed in $N$. maura inhabiting rice fields compared to snakes in natural wetlands, likely because prey are not available in fields and canals until after they are flooded in the spring (Santos and others, 2005). It is possible that the timing of reproduction in giant gartersnakes will vary between sites and among years in response to the timing of rice planting and canal flooding. This would be an interesting future research question to study in giant gartersnakes. 


\section{Summary and Conclusions}

The 4 years of study at the 8 demographic monitoring sites have shown that it is possible to measure important demographic rates in giant gartersnake populations with the amount of sampling effort expended and that greater sampling effort produces more precise estimates. Sites differed in their estimated abundance, survival, and the size distribution of giant gartersnakes in the population. It is also apparent that there is variation in the amount of aquatic habitat available for giant gartersnakes because the amount of active rice growing fluctuated over time and varied among sites. Continued sampling in the future would allow for estimation of temporal trends in abundance and vital rates and for investigation of how the amount of rice grown affects the demography and health of giant gartersnake populations.

- We trapped for giant gartersnakes at eight sites in the Sacramento Valley, California, from 2014-17.

- Trapping in 2014 and 2015 was not extensive and was designed to capture snakes for radio-transmitter implantation as part of an earlier study.

- Sampling effort increased in 2016 and 2017 to capture more snakes for capture-markrecapture analysis and estimation of abundance, growth, survival, and fecundity.

- We made 2,995 captures of 1,011 snakes from 2014-17.

- The majority of snakes were captured for the first time in 2016 or 2017, when trapping effort increased at the start of this study.

- Abundance was highest at site 8 at lowest at sites 5 and 7.

- The area undergoing active rice growing varied among sites and over time.

- The probability of capturing an individual snake at least once during the sampling period increased in 2016 and 2017, the 2 years with full trapping effort designed for capturemark-recapture.

- Estimates of abundance, survival, and population growth rate were more precise in 2016 and 2017 than previous years in which trapping effort was low.

- Both female fecundity (litter size) and the probability a female was gravid exhibited a positive relationship with female snout-vent length. 


\section{References Cited}

Bauwens, D., and Claus, K., 2018, Do newborn adders suffer mass mortality or do they venture in a collective hide-and-seek game?: Biological Journal of the Linnean Society, v. 124, p. 99112.

Brooks, S.P., and Gelman, A., 1998, General methods for monitoring convergence of iterative simulations: Journal of Computational and Graphical Statistics, v. 7, p. 434-455.

Campbell, T.H., and Ellis, C.K., 2007, Avian and exotic animal hematology and cytology (3d ed.): Ames, Iowa, Blackwell Publishing, 286 p.

Casazza, M.L., Wylie, G.D., and Gregory, C.J., 2000, A funnel trap modification for surface collection of aquatic amphibians and reptiles: Herpetological Review, v. 31, p. 91-92.

Denwood, M.J., 2016, runjags: An R package providing interface utilities, model templates, parallel computing methods and additional distributions for MCMC models in JAGS: Journal of Statistical Software, v. 71, p. 1-25.

Fish and Game Commission, 1971, California Code of Regulations: Animals of California Declared to be Endangered or Threatened, Title 14, section 670.5.

Frayer, W.E., Peters, D.D., and Pywell, H.R., 1989, Wetlands of the California Central Valleystatus and trends, 1939 to mid-1980s: Portland, Oreg., U.S. Fish and Wildlife Service, 27 p.

Gelman, A., 2006, Prior distribution for variance parameters in hierarchical models: Bayesian Analysis, v. 1, p. 515-533.

Halstead, B.J., Skalos, S.M., Casazza, M.L., and Wylie, G.D., 2015a, Realized detection and capture probabilities for giant gartersnakes (Thamnophis gigas) using modified floating aquatic funnel traps: U.S. Geological Survey Open-File Report 2015-1200, 36 p., https://doi.org/10.3133/ofr20151200.

Halstead, B.J., Skalos, S.M., Wylie, G.D., and Casazza, M.L., 2015b, Terrestrial ecology of semi-aquatic giant gartersnakes (Thamnophis gigas): Herpetological Conservation and Biology, v. 10, p. 633-644.

Halstead, B.J., Wylie, G.D., and Casazza, M.L., 2010, Habitat suitability and conservation of the giant gartersnake (Thamnophis gigas) in the Sacramento Valley of California: Copeia, v. 2010, p. 591-599.

Halstead, B.J., Wylie, G.D., and Casazza, M.L., 2013, Efficacy of trap modifications for increasing capture rates of aquatic snakes in floating aquatic funnel traps: Herpetological Conservation and Biology, v. 8, p. 65-74.

Halstead, B.J., Wylie, G.D., and Casazza, M.L., 2014, Ghost of habitat past: historic habitat affects the contemporary distribution of giant garter snakes in a modified landscape: Animal Conservation, v. 17, p. 144-153.

Hansen, E.C., Scherer, R.D., Fleishman, E., Dickson, B.G., and Krolick, D., 2017, Relations between environmental attributes and contemporary occupancy of threatened giant gartersnakes (Thamnophis gigas): Journal of Herpetology, v. 51, p. 274-283.

Hansen, E.C., Scherer, R.D., White, G.C., Dickson, B.G., and Fleishman, E., 2015, Estimates of survival probability from two populations of giant gartersnakes in California's Great Central Valley: Copeia, v. 103, p. 1026-1036.

Kéry, M., and Royle, J.A., 2016, Applied hierarchical modeling in ecology: San Diego, Calif., Academic Press, 783 p.

Kéry, M., and Schaub, M., 2012, Bayesian population analysis using WinBUGS: a hierarchical perspective: Waltham, Mass., Academic Press, 554 p. 
Pike, D.A., Pizzatto, L., Pike, B.A., and Shine, R., 2008, Estimating survival rates of uncatchable animals: the myth of high juvenile mortality in reptiles: Ecology, v. 89, p. 607-611.

Plummer, M., 2003, JAGS: A program for analysis of Bayesian graphical models using Gibbs sampling: Proceedings of the 3rd International Workshop on Distributed Statistical Computing (DSC 2003), p. 20-22.

Polson, N.G., and Scott, J.G., 2012, On the half-Cauchy prior for a global scale parameter:

Bayesian Analysis, v. 7, p. 887-902.

R Core Team, 2016, R: a language and environment for statistical computing: Vienna, Austria, $R$ Foundation for Statistical Computing.

Reyes, G.A., Halstead, B.J., Rose, J.P., Ersan, J.S.M., Jordan, A.C., Essert, A.M., Fouts, K.J., Fulton, A.M., Gustafson, K.B., Wack, R.F., Wylie, G.D., and Casazza, M.L., 2017, Behavioral response of giant gartersnakes (Thamnophis gigas) to the relative availability of aquatic habitat on the landscape: U.S. Geological Survey Open-File Report 2017-1141, 134 p., https://doi.org/10.3133/ofr2017114/1.

Rose, J.P., Ersan, J.S.M., Wylie, G.D., Casazza, M.L., and Halstead B.J., 2018a, Construction and analysis of a giant gartersnake (Thamnophis gigas) population projection model: U.S. Geological Survey Open-File Report 2017-1164, 98 p., https://doi.org/10.3133/ofr20171164.

Rose, J.P., Halstead, B.J., Wylie, G.D., and Casazza, M.L., 2018b, Spatial and temporal variability in growth of giant gartersnakes: plasticity, precipitation, and prey: Journal of Herpetology v. 52, p. 40-49.

Rose, J.P., Ersan, J.S.M., Wylie, G.D., Casazza, M.L., and Halstead, B.J., 2018c, Reproductive frequency and size-dependence of fecundity in the giant gartersnake (Thamnophis gigas): Herpetological Conservation and Biology, v. 13, p. 80-90.

Santos, X., Llorente, G.A., Feriche, M., Pleguezuelos, J.M., Casals, F., and de Sostoa, A., 2005, Food availability induces geographic variation in reproductive timing of an aquatic oviparous snake (Natrix maura): Amphibia-Reptilia, v. 26, p. 183-191.

Seigel, R.A., and Ford, N.B, 2001, Phenotypic plasticity in reproductive traits: geographical variation in plasticity in a viviparous snake: Functional Ecology, v. 15, p. 36-42.

U.S. Fish and Wildlife Service, 1993, Endangered and threatened wildlife and plantsDetermination of threatened status for the giant garter snake: Federal Register v. 58, p. 5405354066.

Wack, R.F., Hansen, E., Small, M., Poppenga, R., Bunn, D., and Johnson, C.K., 2012, Hematology and plasma biochemistry values for the giant garter snake (Thamnophis gigas) in the Sacramento Valley of California: Journal of Wildlife Diseases, v. 48, p. 307-313.

Winne, C.T., Willson, J.D., Andrews, K.M., and Reed, R.N., 2006, Efficacy of marking snakes with disposable medical cautery units: Herpetological Review, v. 37, p. 52-54.

Zhong, L., Hongbing, Y., Reyes, E., and Chung, F., 2016, Early-season rice field mapping using Landsat imagery in the Sacramento Valley: California Water and Environmental Modeling Forum, 2016 Annual Meeting Proceedings, p. 2-6. 


\section{Glossary}

Apparent survival ( $\phi)$ : The joint probability that an individual survives and does not permanently leave the study area in a capture-mark-recapture study. Capture-mark-recapture studies, based on marking individuals within a defined study area, estimate apparent survival, not true survival, because it is not possible to know with certainty whether an individual that is not captured for several years died or emigrated offsite. To estimate true survival, additional data, such as the recovery of dead individuals or sighting of emigrants out of the capture-mark-recapture study area, are needed.

Asymptotic size $\left(L_{\infty}\right)$ : The theoretical maximum size an individual approaches as it grows with age in a von Bertalanffy growth model. The asymptotic size describes a population level average, and some individuals may reach maximum sizes greater than the asymptotic size for the population or species.

Bayesian analysis: A method of statistical inference in which Bayes' rule is used to update probability distributions as data are acquired.

Capture-mark-recapture: A method of estimating demographic parameters of populations for which individuals cannot be directly counted that involves capturing, marking, and recapturing individuals. Not all capture-mark-recapture methods require physical capture of individuals (for example, camera traps or hair snares for DNA).

Capture probability: The per-survey probability that a specific individual is captured or observed. Convergence: The meeting and overlapping of independent Markov chains in a Markov Chain Monte Carlo simulation used to assess whether the stationary posterior distribution has been reached.

Covariate: A (predictor) variable thought to be correlated with another (response) variable used in a regression analysis.

Credible interval: A specified domain of the posterior probability distribution used for interval estimation. Credible intervals can be interpreted as the probability, confidence, or belief that a parameter or value falls within the specified region.

Emigration: The movement of individuals outside the sampled area. Emigration can be temporary or permanent.

Fecundity: The number of offspring produced by a female in a given year (litter size).

Gravid: Pregnant or full of eggs.

Informative prior: A prior probability distribution that expresses prior information or knowledge about a variable based on data from previous studies or expert knowledge.

Jolly-Seber model: An open capture-mark-recapture model that simultaneously estimates survival between sampling periods and the abundance of the population during each sampling period. The Jolly-Seber model attempts to estimate how many individuals were present in the population but not captured during each sampling period.

Linear: Involving the effect of a predictor variable that is raised to the first power in a regression model.

Litter: The number of young brought forth by an animal with multiple reproductive cycles at one parturition.

Markov Chain Monte Carlo (MCMC): A class of algorithms for sampling from a probability distribution that is based on constructing a Markov chain that has the desired distribution as its equilibrium distribution.

Neonate: A newborn.

Parturition: The act or process of giving birth to offspring. 
Population growth rate $(\lambda)$ : The change in abundance of a population in year $t+1$ relative to year $t$. A value of $\lambda=1$ represents a stable population (no change in abundance), $\lambda$ less than 1 indicates a declining population, and $\lambda$ greater than 1 indicates an increasing population. $\lambda$ can be calculated from a time series of abundances, for example, $\lambda=N_{t+1} / N_{t}$.

Posterior distribution: The probability distribution of an unknown quantity conditional on the evidence obtained from an experiment or survey, possibly taking into account relevant information available prior to the experiment or survey.

Posterior probability: The conditional probability assigned to a random event after the relevant evidence or background has been taken into account.

Precinctive: Restricted to a defined geographic area.

Prior probability: The probability distribution that expresses uncertainty about an unknown quantity before some evidence (experiment, survey, observation) is taken into account.

Quadratic: Involving the square of a predictor variable in a regression model.

Recruitment: The process by which offspring born in year $t$ survive and grow to join the censused population in year $t+1$. Only a fraction of the neonates produced in year $t$ will survive to recruit to the censused population in year $t+1$

Somatic growth: The rate of change in an individual animal's body size (for example, length). Snout-vent length (SVL): A measure of body length commonly used in reptiles. Length is measured from the anterior tip of the snout to the vent or opening of the cloaca. SVL is generally preferred over total body length because in reptiles many individuals lose parts of their tail during their life (likely from attempted predation); thus, SVL is comparable among individuals. Survival: The rate or probability at which individuals alive at time $t$ remain alive until time $t+1$. Uninformative prior: A prior probability distribution that expresses vague or general information about a variable, typically with the goal of assigning equal probabilities to all possibilities. Vital rate: Also called a demographic rate; the rates of survival, growth, and reproduction in a population. Vital rates are often linked to individual states or characteristics, such as size, age, or sex, to allow researchers to simulate the way in which the population will change over time. von Bertalanffy growth model: A model of growth in body size where individuals initially increase rapidly in size. Then growth slows as individuals approach an asymptotic size. This model is commonly used to describe the growth of fish, reptiles, and other ectotherms. 


\section{Appendix A. Details of Bayesian Models}

The models of female reproductive state (gravid or not) and fecundity were each run on five independent chains. The five chains were run for 50,000 iterations each after an initial burnin of 1,000 iterations. The resulting chains were thinned by a factor of 10 , resulting in 5,000 iterations per chain and a total posterior sample of 25,000 .

The Jolly-Seber open-population capture-mark-recapture model was run on four independent chains for 75,000 iterations each, following an initial burn-in of 10,000 iterations. The resulting chains were thinned by a factor of three, resulting in 25,000 iterations per chain, and a total posterior sample of 100,000.

The von Bertalanffy growth model was run on five independent chains for 30,000 iterations each after an initial burn-in of 3,000 iterations. The model of giant gartersnake growth rates was run on five independent chains for 1,000,000 iterations each, after an initial burn-in of 10,000 iterations. The chains were thinned by 500, resulting in 2,000 samples per chain, and a total posterior of 10,000 samples.

Table A1. Definition of priors for parameters in the reproductive state model. This model estimates the relationship between reproductive state and size (snout-vent length) for female giant gartersnakes from eight sites in the Sacramento Valley, California, in 2017.

$[\mathrm{SD}=$ standard deviation; $\mathrm{SVL}=$ snout-vent length $]$

\begin{tabular}{llll}
\hline \multicolumn{1}{c}{ Parameter } & Symbol & Prior distribution \\
\hline Mean probability a female is gravid & $\mathrm{p}_{\mathrm{g}}$ & Uniform $(0,1)$ \\
Linear effect of maternal SVL on reproductive status & $\beta g_{\mathrm{SVL}}$ & Normal $(0,10)$ \\
Linear effect of exam date on reproductive status & $\beta_{\text {date } 1}$ & Normal $(0,10)$ \\
Quadratic effect of exam date on reproductive status & $\beta_{\text {date2 }}$ & Normal $(0,10)$ \\
SD of site random effect on $\alpha$ & $\sigma_{\text {site }}$ & Half-Cauchy $(0,1)$ \\
\hline
\end{tabular}

Table A2. Definition of priors for parameters in the fecundity model. This model estimates the relationship between size (snout-vent length) and fecundity for female giant gartersnakes from eight sites in the Sacramento Valley, California, in 2017.

$[\mathrm{SD}=$ standard deviation; $\mathrm{SVL}=$ snout-vent length $]$

\begin{tabular}{lll}
\hline \multicolumn{1}{c}{ Parameter } & \multicolumn{1}{c}{ Symbol } & Prior distribution \\
& & \\
\hline Intercept of maternal size-fecundity relationship & $\alpha_{\mathrm{f}}$ & Normal $(0,10)$ \\
Linear effect of maternal SVL on litter size & $\beta \mathrm{f}_{\mathrm{SVL}}$ & $\operatorname{Normal}(0,10)$ \\
SD of site random effect on $\alpha$ & $\sigma_{\text {site }}$ & Half-Cauchy $(0,1)$ \\
\hline
\end{tabular}


Table A3. Definition of priors for parameters in the Jolly-Seber model. This model estimates abundance and survival from capture-mark-recapture data of giant gartersnakes at eight sites in the Sacramento Valley, California, from 2014-17.

$[\mathrm{SD}=$ standard deviation $]$

\begin{tabular}{|c|c|c|}
\hline Parameter & Symbol & Prior distribution \\
\hline Mean daily capture probability & $p$ & Uniform $(0,1)$ \\
\hline Mean apparent survival probability & $\phi$ & Uniform $(0,1)$ \\
\hline $\mathrm{SD}$ of site random effect on $p$ & $\sigma_{p, \text { site }}$ & Half-Cauchy $(0,1)$ \\
\hline SD of year random effect on $p$ & $\sigma_{p, \mathrm{t}}$ & Half-Cauchy $(0,1)$ \\
\hline SD of individual random effect on $p$ & $\sigma_{p, \text { ind }}$ & Half-Cauchy $(0,1)$ \\
\hline SD of site random effect on $\phi$ & $\sigma_{\phi, \text { site }}$ & Half-Cauchy $(0,1)$ \\
\hline SD of year random effect on $\phi$ & $\sigma_{\phi, t}$ & Half-Cauchy $(0,1)$ \\
\hline Entrance probability & $\eta$ & $\operatorname{Gamma}(1,1)$ \\
\hline
\end{tabular}


Table A4. Definition of priors for parameters in the growth models. This includes a von Bertalanffy growth model and a model of growth rates (in $\mathrm{mm} / \mathrm{day}$ ) for giant gartersnakes at eight sites in the Sacramento Valley, California, from 2014-17.

[SD = standard deviation; $\mathrm{SVL}=$ snout-vent length $]$

\begin{tabular}{|c|c|c|c|}
\hline Model & Parameter & Symbol & Prior distribution \\
\hline \multirow{10}{*}{$\begin{array}{l}\text { von } \\
\text { Bertalanffy } \\
\text { growth }\end{array}$} & Mean asymptotic size (mm) & $\mathrm{L}_{\infty}$ & Normal $(1,000,100)$ \\
\hline & Mean growth coefficient & $k$ & Uniform $(0,0.01)$ \\
\hline & Individual variability in growth & $\lambda$ & Uniform $(0,100)$ \\
\hline & SD of year random effect on $k$ & $\sigma_{\mathrm{ky}}$ & Uniform $(0,0.01)$ \\
\hline & SD of site random effect on $k$ & $\sigma_{\mathrm{ks}}$ & Uniform $(0,0.01)$ \\
\hline & SD of site random effect on $\mathrm{L}_{\infty}$ & $\sigma_{\mathrm{L}}$ & Uniform $(0,200)$ \\
\hline & SD of measurement error & $\sigma_{\mathrm{e}}$ & $\operatorname{Gamma}(0.001,0.001)$ \\
\hline & Sex effect on $\mathrm{L}_{\infty}(\mathrm{mm})$ & $\mathrm{m}_{\mathrm{L}}$ & $\operatorname{Normal}(0,316)$ \\
\hline & Sex effect on $k$ & $\mathrm{~m}_{\mathrm{k}}$ & $\operatorname{Normal}(0,0.01)$ \\
\hline & Sex effect on $\lambda$ & $\mathrm{m}_{\lambda}$ & $\operatorname{Normal}(0,100)$ \\
\hline \multirow[t]{8}{*}{ Growth rate } & Mean growth rate & $\alpha_{\mathrm{GR}}$ & $\operatorname{Normal}(0,1)$ \\
\hline & Effect of sex on growth rate & $\beta_{\text {sex }}$ & $\operatorname{Normal}(0,1)$ \\
\hline & Effect of SVL on growth rate & $\beta_{\mathrm{SVL}}$ & $\operatorname{Normal}(0,1)$ \\
\hline & Effect of sex $\times$ SVL interaction on growth rate & $\beta_{\mathrm{sex} \times \mathrm{SVL}}$ & $\operatorname{Normal}(0,1)$ \\
\hline & SD of residual variation in growth rate & $\sigma_{\mathrm{GR}}$ & Half-Cauchy $(0,1)$ \\
\hline & SD of year random effect on $\alpha_{\mathrm{GR}}$ & $\sigma_{\text {site }}$ & Half-Cauchy $(0,1)$ \\
\hline & $\mathrm{SD}$ of site random effect on $\alpha_{\mathrm{GR}}$ & $\sigma_{\text {year }}$ & Half-Cauchy $(0,1)$ \\
\hline & SD of individual random effect on $\alpha_{\mathrm{GR}}$ & $\sigma_{\text {ind }}$ & $\operatorname{Half-Cauchy}(0,1)$ \\
\hline
\end{tabular}


Publishing support provided by the U.S. Geological Survey Science Publishing Network, Sacramento Publishing Service Center

For more information concerning the research of this report, contact the Director, California Water Science Center U.S. Geological Survey 6000 J Street, Placer Hall

Sacramento, California 95819

http://ca.water.usgs.gov 
